\title{
Stability analysis for the background equations for inflation with dissipation and in a viscous radiation bath
}

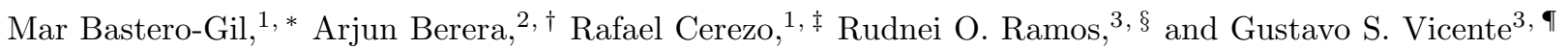 \\ ${ }^{1}$ Departamento de Física Teórica y del Cosmos, Universidad de Granada, Granada-18071, Spain \\ ${ }^{2}$ SUPA, School of Physics and Astronomy, University of Edinburgh, Edinburgh, EH9 3JZ, United Kingdom \\ ${ }^{3}$ Departamento de Física Teórica, Universidade do Estado do Rio de Janeiro, 20550-013 Rio de Janeiro, RJ, Brazil
}

\begin{abstract}
The effects of bulk viscosity are examined for inflationary dynamics in which dissipation and thermalization are present. A complete stability analysis is done for the background inflaton evolution equations, which includes both inflaton dissipation and radiation bulk viscous effects. Three representative approaches of bulk viscous irreversible thermodynamics are analyzed: the Eckart noncausal theory, the linear and causal theory of Israel-Stewart and a more recent nonlinear and causal bulk viscous theory. It is found that the causal theories allow for larger bulk viscosities before encountering an instability in comparison to the noncausal Eckart theory. It is also shown that the causal theories tend to suppress the radiation production due to bulk viscous pressure, because of the presence of relaxation effects implicit in these theories. Bulk viscosity coefficients derived from quantum field theory are applied to warm inflation model building and an analysis is made of the effects to the duration of inflation. The treatment of bulk pressure would also be relevant to the reheating phase after inflation in cold inflation dynamics and during the radiation dominated regime, although very little work in both areas has been done; the methodology developed in this paper could be extended to apply to these other problems.
\end{abstract}

PACS numbers: $98.80 . \mathrm{Cq}$

\section{INTRODUCTION}

Cosmological inflationary models are usually described by the evolution of a background scalar field, the inflaton. These models can be separated in terms of isentropic (cold) [1, 2] and nonisentropic (warm) [3, 4] expansion (for earlier related work see [5]), when regarding the production of radiation during the inflation phase. In cold inflation (CI), the couplings of the inflaton field to other field degrees of freedom are considered not to influence the inflaton dynamics in any appreciable way, becoming only relevant at the end of inflation, at the time of reheating. As such, in cold inflation dynamics, during inflation the universe undergoes a stage of supercooling. In contrast for warm inflation (WI), the interactions of the inflaton field with other field degrees of freedom are strong enough to lead to radiation production at a rate that can eventually compensate the supercooling due to expansion. This will lead to some general statistical state during inflation.

The analysis of the quantum dynamics of inflationary models is done using the effective action with the in-in Schwinger closed-time path (or Schwinger-Keldysh) method [6]. This approach has been used for the study of the quantum dynamics of warm inflation since its early implementations [7]. A review of these quantum field theory methods applied to warm inflation can be found e.g. in Ref. [8]. In the context of cold inflation, the SchwingerKeldysh method is also commonly used and one primary modification it makes to the tree level theory is to add time local corrections to the effective potential due to radiative effects. Another modification that can be studied by this method is the growth of long wavelength quantum fluctuations and their effect on the evolution of the inflaton background and fluctuation modes [9]. Warm inflation models are those in which significant time nonlocal contributions also arise from the effective action. Main consequences of such terms will be dissipation and fluctuation effects [6, 10]. This can very generally lead to a vast variety of particle production behavior during inflation, thus to various types of statistical states. Amongst all of these, the one case which can be studied most extensively by quantum field theory methods is the case where the statistical state of the Universe emerging from these effects is near thermal equilibrium. Once particle production occurs during inflation, there are many effects that can follow and it is a complicated problem. This is why it is essential to understand the properties of warm inflation in the near

\footnotetext{
*Electronic address: mbg@ugr.es

${ }^{\dagger}$ Electronic address: ab@ph.ed.ac.uk

‡Electronic address: cerezo@ugr.es

$\S$ Electronic address: rudnei@uerj.br

IElectronic address: gsvicente@uerj.br
} 
thermal equilibrium regime, so as to provide insight into the features that one should also expect when away from this limit. Of late there have been some efforts to understand warm inflation models away from thermal equilibrium [11]. These papers have analyzed some of the effects that emerge in the presence of particle production, but they are at a much more primary level then the extensive study for the near thermal equilibrium regime. In particular, creating a radiation bath of particles will also then modify the inflaton potential no matter what statistical state it is in. Also the nature of the radiation bath of particles will alter and modify the density perturbations. In the thermal limit there are well established methods for examining all these effects, whereas away from this limit it is a much more difficult problem.

As mentioned above, the case that has been studied most extensively, since it is the most tractable by quantum field theory methods, is when the radiation has adequate time to reach close to a thermal state. For this case, the radiation production during inflation manifests itself through an effective dissipation term in the inflaton background equation. The corresponding dissipative coefficient can be computed from first principles in quantum field theory under appropriate conditions (see e.g. Refs. 7, 8, 12 15]). In particular, the two-stage interaction configuration for quantum fields proposed in [16, 17], has been shown can lead to a large enough dissipative terms, while keeping the quantum corrections to the inflationary potential under control and allowing a period of slow-roll inflation [18 [20].

In WI, the transfer of energy of the inflaton field to the radiation bath is mediated by the dissipation term in the inflaton's evolution equation. Nevertheless, an additional effect can arise due to inner couplings in the radiation fluid itself. Internal decays within the radiation fluid can make it depart slightly from thermal equilibrium. Therefore, the radiation fluid can behave as a non-ideal fluid and viscosity effects must be taken into account [21]. At the background level, the relevant viscous effect is due to bulk pressure, since it is the only viscous effect appearing in the background equations for an FRW universe.

The study of bulk viscous effects in cosmology, and in particular in inflation, has some history to it, focused mainly on the effect of the bulk pressure as a negative pressure (for a partial sample of the earlier works on bulk viscous cosmologies, see for example Ref. [22]). In addition, more recently, there has been a surge of interest in exploring the effects of the bulk pressure as the origin of the present accelerated expansion of the universe (see e.g. Refs. [23]). Almost all of these past works only use phenomenological forms for the bulk viscosity. This paper differs from these past works since we will apply first principle quantum field theory computed expressions for the bulk viscosity, based on the calculations found e.g. in Ref. [24], to warm inflation. There has been very little work done in studying first principles bulk viscosity expressions in application to cosmology. There is one paper we are aware of along these lines, which is the first reference in [23]; this paper uses quantum field theory derived expressions of bulk viscosity and shows how they can play the role similar to dark energy.

Our work will examine the consequences of bulk viscosity on warm inflationary dynamics. In particular we are interested in determining the stability conditions of the background equations of warm inflation when coupled to the bulk viscous radiation bath. Earlier studies examining the stability properties of the warm inflation equations include Refs. [20, 25], but these studies did not include the effects of bulk viscous pressure. Preliminary studies on the inclusion of bulk viscous pressure have been done in [26], 27], where only the non-causal theory of Eckart [28] has been used. Here we extend the stability analysis of the dynamical warm inflation equations to include bulk viscous effects. Moreover our analysis will be done not just for the non-causal Eckart theory [28] but also the causal theories of bulk pressure 29 31], with a full analysis of the differences in the resulting dynamics from these different theories.

In the study of viscous effects in cosmology it is common to use linear expressions to describe the viscous pressure, where it is assumed that the deviations are close to equilibrium. However, the viscous pressure can, in principle, take the system far from its thermodynamical equilibrium, so, we must apply suitable approaches in order to see if one really needs to use a more robust description, incorporating nonlinear effects. We consider here three different theories to describe the viscous pressure: the non-causal theory due to Eckart [28], the linear and causal theory of Israel-Stewart [29, 30] and finally we will also use a recent causal and nonlinear theory proposed by the authors in 31], named by them Nonlinear Causal Dissipative Hydrodynamics (NLCDH). There have been other proposals for a nonlinear theory for the bulk viscous pressure 32, 33, that make use of ad-hoc parameters, such as the time where nonlinear effects become important [32] or functions [33] that do not have an immediate interpretation from quantum field theory, for example being a relaxation time. In using such approaches there is no immediate understanding how to associate their parameters with first principles parameters. We have considered the theory for bulk pressure in [31] since it utilizes parameters which can readily be determined from microscopic physics, in particular from quantum field theory. The theories for the bulk pressure we analyze here are more natural to use in field theory model building, where the dissipation terms, viscosity coefficients and relaxation times are well defined and can be reliably computed once a specific field theory model is given.

We study the effects of the inclusion of bulk viscosity in three commonly used supersymmetric realizations of warm inflation, the chaotic, hybrid and hilltop models. As the bulk viscosity modifies the background dynamics of warm inflation, it also changes the available parameter space for warm inflation, which is analyzed here. The bulk viscosity is approximated by the Eckart description and we place limits on the couplings of the underlying particle physics 
theory for the validity of this approximation.

The paper is organized as follows. In Sec. [II we summarize the relevant expressions for warm inflation and for the theories of bulk viscous pressure that is relevant for this work. In Sec. III we present the respective dynamical systems in each of the three theories for the bulk viscous pressure we are examining, along with the resulting stability equations. In Sec. IV] we present some numerical results that confirm the stability results. Also in this section there is an exposition about the differences in the dynamics that emerge when considering noncausal and causal theories for the bulk pressure. In Sec. $\mathrm{V}$ we discuss quantum field theory derived expressions for the bulk viscosity and conditions under which they can be applied to cosmology. In Sec. VI we apply the stability analyses to explore the parameter space available for warm inflation constructed from supersymmetric models. Finally, in Sec. VII] we present our conclusions and final comments.

\section{WARM INFLATION IN A BULK VISCOUS RADIATION FLUID}

In the warm inflation scenario, the inflaton evolution is described in terms of a dissipative equation of motion of the form $[\underline{3}, 4,4,12]$

$$
\ddot{\phi}+(3 H+\Upsilon) \dot{\phi}+V_{, \phi}=0,
$$

where $\Upsilon$ is the dissipative coefficient, $V_{, \phi}$ is the field derivative of the (field and temperature dependent) effective potential, $H=\dot{a} / a$ is the Hubble rate of expansion, and $a$ the scale factor of the Friedmann-Robertson-Walker background metric, $d s^{2}=-d t^{2}+a(t)^{2} \delta_{i j} d x^{i} d x^{j}$.

The dissipation term in Eq. (2.1) originates from the interactions of the inflaton field with other fields. These interactions effectively render the inflaton as an open system, which necessarily should give origin to dissipation. The dissipation coefficient $\Upsilon$ accounts for this effective dissipation of the inflaton and, given a particle physics model realization of inflation, it can be computed microscopically (see e.g. the recent review [8] and references there in). If enough dissipation, i.e., radiation, can be produced during the inflationary regime, it can eventually compensate the redshift of the radiation due to expansion. When this happens, we then term the inflationary regime as warm inflation. A particular field theory model that has been proved successful in generating the WI conditions is the two-stage mechanism [16 18]. In the two-stage mechanism, the inflaton field couples to a heavy catalyst field, and the latter in turn couples to light degrees of freedom. The evolution of the background inflaton field excites the catalyst fields, which then decay into light fields. Large dissipation has been demonstrated with this simple mechanism, adequate to drive a warm inflationary expansion. When the produced light relativistic particles thermalize, which is achieved when their creation and scattering times are less than the Hubble time in an expanding universe, we can model their contribution as that of radiation,

$$
\rho_{r} \simeq \frac{\pi^{2}}{30} g_{*} T^{4},
$$

where $T$ is the temperature of the thermal bath, and $g_{*}$ the effective number of light degrees of freedom ${ }^{1}$.

Bulk viscous effects come in to play when the light particles in the resulting radiation bath in warm inflation can also decay, thus making the radiation fluid slightly depart from equilibrium. This effectively renders the radiation as a nonideal fluid and gives origin to a bulk viscous pressure (there may be other dissipative effects in the radiation fluid itself, like shear viscous stresses, but these do not contribute at the background level [21], though they may be relevant in the determination of the spectrum of density perturbations, as demonstrated recently [34]). In the presence of a bulk viscous pressure, $\Pi$, the stress-energy tensor for the radiation fluid is given by [21, 35],

$$
\mathcal{T}_{\mu \nu}^{(r)}=\left(\rho_{r}+p_{r}+\Pi\right) u_{\mu}^{(r)} u_{\nu}^{(r)}+\left(p_{r}+\Pi\right) g_{\mu \nu},
$$

where $\rho_{r}$ is the radiation energy density, $p_{r}$ the adiabatic radiation pressure, $u_{\mu}^{(r)}$ are the four velocity of the radiation fluid and $g_{\mu \nu}$ the four-dimensional metric. It happens then that the bulk pressure enters as a contribution to the radiation pressure $p_{r}$, such that we can define in general an effective pressure for the radiation, $\tilde{p}_{r}$, given by

\footnotetext{
${ }^{1}$ If not otherwise specified, we will take $g_{*}=228.75$, the effective no. of degrees of freedom for the Minimal Supersymmetric Standard Model, when presenting numerical results.
} 


$$
\tilde{p}_{r}=p_{r}+\Pi .
$$

The evolution equation for the radiation fluid energy density $\rho_{r}$ then becomes

$$
\dot{\rho}_{r}+3 H\left(\rho_{r}+\tilde{p}_{r}\right)=\Upsilon\left(\rho_{\phi}+p_{\phi}\right),
$$

where $p_{\phi}=\dot{\phi}^{2} / 2-V(\phi, T)$, and $\rho_{\phi}+p_{\phi}=\dot{\phi}^{2}$.

It is also useful to express this in terms of the entropy density $s$. From the Helmholtz free energy $f=\rho-T s$, where $f=V(\phi, T)$, and using $s=-\partial f / \partial T$, the total energy density $\rho$ becomes

$$
\rho=\frac{\dot{\phi}^{2}}{2}+V(\phi, T)+T s,
$$

and the Hubble rate $H$ becomes

$$
H^{2}=\frac{1}{3 m_{\mathrm{P}}^{2}}\left[\frac{\dot{\phi}^{2}}{2}+V(\phi, T)+T s\right],
$$

where $m_{\mathrm{P}}$ is the reduced Planck mass, $m_{P}=1 / \sqrt{8 \pi G}=2.4 \times 10^{18} \mathrm{GeV}$. Using also that $p_{r}=(\gamma-1) \rho_{r}$ and that the entropy density $s$ is related to the radiation energy density by $T s=\gamma \rho_{r}$, Eq. (2.5) can then be written in terms of the entropy density as

$$
T \dot{s}+3 H(T s+\Pi)=\Upsilon \dot{\phi}^{2},
$$

where we have used $\gamma=4 / 3$, which is valid for a quasi-equilibrium high temperature thermal bath typical of warm inflation.

From Eq. (2.8), the dynamical effects of the bulk viscosity can be easily read. Given that the bulk viscous pressure $\Pi$ is negative, it acts to decrease the radiation pressure, thus enhancing the effect from the source term on the RHS in the equation for the entropy density. As a consequence, the entropy density increases, and therefore the radiation energy density also grows. On the one hand, if this bulk pressure term is too large, there is too much radiation production and the radiation energy density dominates too soon over the scalar field energy density, thus spoiling inflation. This regime is called the unstable regime. On the other hand, if the bulk pressure term is controlled to avoid the radiation domination until the end of inflation, the system is said to be in the stable regime. In this regime the bulk viscosity gives rise to an additional negative pressure, and hence, inflation is enhanced.

To account for the dynamics involving the bulk viscous pressure $\Pi$, as explained in the introduction, we will consider three different theories: the noncausal theory due to Eckart [28], the linear and causal theory of Israel-Stewart (IS) [29, 30], and a recent causal and nonlinear theory, Nonlinear Causal Dissipative Hydrodynamics (NLCDH), proposed in [31]. The starting point to build these hydrodynamic theories is the conservation equations of the stressenergy tensor and the number density vector $N^{\mu}=n u^{\mu}$,

$$
\nabla_{\mu} \mathcal{T}^{\mu \nu}=0, \quad \nabla_{\mu} N^{\mu}=0,
$$

with the additional condition on the 4-entropy, written in terms of the entropy density $s, s^{\mu}=s u^{\mu}$, that must satisfy the second law of thermodynamics in its covariant form,

$$
\nabla_{\mu} s^{\mu} \geq 0 .
$$

The 4-entropy, just like the stress-energy tensor, gains a contribution coming from the dissipative fluxes,

$$
s^{\mu}=s u^{\mu}+\frac{Q^{\mu}}{T},
$$

where $Q^{\mu}=Q^{\mu}\left(N^{\mu}, \mathcal{T}^{\mu \nu}\right)$ accounts for the dissipative fluxes. The irreversible thermodynamics comprises of the dissipative forces to the hydrodynamics variables at equilibrium, the number density $n$, the energy density $\rho$ and the pressure $p$. These quantities are able to describe the energy fluxes in a nonideal fluid. There are different ways in which this can be done, which lead to different descriptions for the dissipative fluxes, like for example for the bulk pressure. We summarize below the Eckart, IS and NLCDH theories for the bulk pressure. 


\section{A. Eckart theory for the bulk pressure}

The Eckart theory [28] assumes that the entropy vector $s^{\mu}$ is linear in the dissipative fluxes. The nonequilibrium contribution to the entropy vector, $Q^{\mu}$, to first order, should then be proportional to the dissipative fluxes. Neglecting dissipative terms other than the bulk pressure, we then have that

$$
Q^{\mu} \simeq a(n, \rho) \Pi u^{\mu}
$$

where the proportionality factor is obtained from the equilibrium condition and from the covariant form of the second law of thermodynamics, Eq. (2.10). This then gives [35]

$$
T \nabla_{\mu} s^{\mu} \simeq-3 H \Pi
$$

To ensure that the second law of thermodynamics, Eq. (2.10), is satisfied and interpreting the term $3 H$ in Eq. (2.13) as a dissipative force, $\chi_{E}=3 H$, we impose $\Pi$ to be linear in this force, expressing bulk viscosity as

$$
\Pi=-3 \zeta_{b} H
$$

where the proportionality term $\zeta_{b} \equiv \zeta_{b}(n, \rho) \geq 0$ is the bulk viscosity coefficient [21].

The bulk pressure expressed like Eq. (2.14) is a noncausal theory, i.e., the speed of the fluxes propagation is infinite. The Eckart theory can be considered in some circumstances as a reasonable approximation for the irreversible thermodynamics. This may happen, for example, when sufficiently short relaxation time scales are involved, otherwise a causal theory would be a much better choice. We now turn to the simplest of such a causal theory, the IS one.

\section{B. Israel-Stewart theory for the bulk pressure}

The IS theory [29, 30] goes one step further than the Eckart theory by accounting for second order contributions beyond equilibrium, by expanding the entropy vector to second order in the dissipative fluxes. Generically this gives, by again only considering the bulk pressure contribution,

$$
s^{\mu} \simeq s u^{\mu}-\beta_{0} \Pi^{2} \frac{u^{\mu}}{2 T}
$$

where $\beta_{0}(n, \rho) \geq 0$. From the covariant derivative of the entropy vector,

$$
T \nabla_{\mu} s^{\mu}=-\Pi\left[3 H+\beta_{0} \dot{\Pi}+\frac{T}{2} \nabla_{\mu}\left(\frac{\beta_{0}}{T} u^{\mu}\right) \Pi\right],
$$

and from the second law of thermodynamics to be satisfied, Eq. (2.10), it is imposed again, like in the Eckart case, that the dissipative fluxes be linear in the dissipative forces. For the bulk pressure $\Pi$ this implies from Eq. (2.16) that

$$
\Pi=-\zeta_{b}\left[3 H+\beta_{0} \dot{\Pi}+\frac{T}{2} \nabla_{\mu}\left(\frac{\beta_{0}}{T} u^{\mu}\right) \Pi\right] .
$$

The relation (2.17) is analogous to Eq. (2.14) in the Eckart theory. The difference here being that, from Eq. (2.13), the Israel-Stewart force is expressed as

$$
\chi_{I S}=3 H+\beta_{0} \dot{\Pi}+\frac{T}{2} \nabla_{\mu}\left(\frac{\beta_{0}}{T} u^{\mu}\right) \Pi .
$$

By defining $\tau=\zeta_{b} \beta_{0}$, which is interpreted as a relaxation time for the bulk viscous processes in the radiation fluid, then Eq. 2.17) can also be rewritten in the form 


$$
\tau \dot{\Pi}+\Pi=-3 \zeta_{b} H-\frac{\zeta_{b} T}{2} \nabla_{\mu}\left(\frac{\tau}{\zeta_{b} T} u^{\mu}\right) \Pi,
$$

and by expanding the derivative in the last term in Eq. (2.19) it can finally be expressed as

$$
\tau \dot{\Pi}+\Pi=-3 \zeta_{b} H-\frac{\tau \Pi}{2}\left(3 H+\frac{\dot{\tau}}{\tau}-\frac{\dot{\zeta}_{b}}{\zeta_{b}}-\frac{\dot{T}}{T}\right),
$$

which is the IS equation for the bulk pressure.

As shown in Ref. [36], the propagation speed for the bulk pressure is given by

$$
c_{\mathrm{visc}}^{2}=\frac{\zeta_{b}}{(\rho+p) \tau}
$$

and, thus, for $\tau \neq 0$ there is a finite propagation speed for the flux, while for the Eckart theory, where $\tau=0$, it is infinity (noncausal). In a quantum field theory description for the radiation bath, e.g. for example in the two-stage decay mechanism mentioned earlier for warm inflation, both the bulk viscosity coefficient $\zeta_{b}$ and the relaxation time $\tau$ can be defined unambiguously and be computed microscopically, just like the dissipation coefficient $\Upsilon$. In particular, the bulk viscosity coefficient can be obtained from a Kubo formula for the high-temperature light particles of the radiation bath [24], and $\tau$ can be associated with the respective decay time of these particles, $\tau=1 / \Gamma$, where $\Gamma$ is the decay width. For the validity of considering a quasi-equilibrium thermal radiation bath, we are then required to impose that

$$
\tau H \equiv H / \Gamma<1 .
$$

Likewise, the assumption of proximity with thermal equilibrium requires the dissipative fluxes to be small compared to the equilibrium pressure,

$$
|\Pi| \ll p .
$$

In terms of the quasi-equilibrium conditions (2.22) and (2.23), the equation for the bulk pressure can also be expressed in terms of the Maxwell-Cattaneo equation for П [37],

$$
\tau \dot{\Pi}+\Pi=-3 \zeta_{b} H .
$$

The IS equation for $\Pi$, Eq. (2.20), can then be seen to give a correction to the Maxwell-Cattaneo equation, which, after Eckart, are the simplest equations for the bulk pressure including relaxation (causal) effects.

\section{Nonlinear causal dissipative hydrodynamics theory for the bulk pressure}

Next, let us consider the NLCDH theory proposed by the authors of Ref. 31]. This theory assumes the Eckart force term, $\chi_{E}=\nabla_{\mu} u^{\mu}=3 H$, plus a memory effect, so as to respect causality. Since this theory in principle makes no assumptions about the linearity of the bulk pressure, as it was assumed in the IS theory for instance, it has been regarded as a nonlinear theory for the bulk pressure. The memory effect adds a relaxation to the system. Recall that the Maxwell-Cattaneo theory Eq. (2.24) is obtained by adding a relaxation time directly to $\Pi$. In the NLCDH instead, the memory effect is added to the quantity $\tilde{\Pi}=\Pi \mathcal{V}$ (where $\mathcal{V}$ is the volume), which is then integrated in a cell (volume element) of the fluid. This is done by imposing the relation $\tilde{\Pi}=-\zeta_{b} \nu_{\chi_{E}}$. Through the addition of the memory effect, we are lead to [31]

$$
\tau \dot{\tilde{\Pi}}+\tilde{\Pi}=-3 H \zeta_{b} \nu
$$

The first term in the above equation results in $\tau(\dot{\Pi} \mathcal{V}+\Pi \dot{\mathcal{V}})$. After using the conservation law for the volume in a cell of the fluid, $\nabla_{\mu}\left(u^{\mu} / \mathcal{V}\right)=0$, it can be shown that [31] 


$$
\nabla_{\mu} \frac{u^{\mu}}{\mathcal{V}}=\left(\frac{1}{\mathcal{V}}\right)+\frac{1}{\mathcal{V}} \nabla_{\mu} u^{\mu}=-\left(\frac{1}{\mathcal{V}}\right)^{2} \dot{\mathcal{V}}+\frac{1}{\mathcal{V}} \chi=0 .
$$

It follows that $\chi=\dot{\mathcal{V}} / \mathcal{V}$ and then $\dot{\mathcal{V}}=\chi \mathcal{V}=3 H \mathcal{V}$. From Eq. (2.25), it then follows the NLCDH equation for the bulk pressure [31],

$$
\tau \dot{\Pi}+\Pi=-3 H\left(\zeta_{b}+\tau \Pi\right) .
$$

\section{THE DYNAMICAL SYSTEM OF EQUATIONS FOR WARM INFLATION IN A BULK VISCOUS RADIATION FLUID}

The relevant equations concerning warm inflation in a bulk viscous radiation fluid are given by the inflaton evolution equation, Eq. (2.1), the entropy energy density evolution Eq. (2.8), with the bulk pressure $\Pi$ given by: (a) in the Eckart case Eq. (2.14); (b) in the IS case by the evolution equation (2.20); and (c) in the NLCDH case by the evolution equation (2.27).

Writing the inflaton equation of motion as two first order differential equations, we have that Eqs. (2.1) and (2.8) are equivalently written in the form:

$$
\begin{aligned}
\dot{\phi} & =u, \\
\dot{u} & =-3 H u-\Upsilon u-V_{, \phi}, \\
T \dot{s} & =-3 H T s-3 H \Pi+\Upsilon u^{2},
\end{aligned}
$$

where

$$
H^{2}=\frac{1}{3 m_{\mathrm{P}}^{2}}\left(\frac{u^{2}}{2}+V+T s\right), \quad V=V(\phi, T), \quad \Upsilon=\Upsilon(\phi, T),
$$

with expression for the bulk pressure $\Pi$, given by either Eq. (2.14), (2.20) or (2.27), depending on which of the cases is treated. In all the cases, they depend on the bulk viscosity coefficient, $\zeta_{b} \equiv \zeta_{b}(T)$. In warm inflation the expected dependence of the dissipation coefficient on the field and temperature is [8, 13, 15]

$$
\Upsilon=C_{\phi} \frac{T^{c}}{\phi^{c-1}},
$$

with proportionality factor $C_{\phi}$ depending on the field content of the model and the value of the power $c$ depending on the temperature regime for the different fields involved. For example, in the two-stage decay model for warm inflation, for a radiation bath of light particles $y$ in the high temperature regime, $m_{y} \ll T$, and for heavy catalyst fields $\chi$ in the low temperature regime, $m_{\chi}>T$, we find that $c=3[8,13,20]$. Likewise, the bulk viscosity coefficient, in this same regime is 24] $\zeta_{b} \propto T^{3}$. In the following we consider a generic power dependence $l$ in the temperature for the bulk viscosity coefficient, $\zeta_{b} \propto T^{l}$, similar to that considered for the dissipation coefficient, Eq. (3.3).

Treating the variables of the dynamical system in the form of a column matrix $\mathbb{X}$, we can express the dynamical system in the compact matrix form,

$$
\dot{\mathbb{X}}=\mathbb{F}(x) \mathbb{X},
$$

where for example, for the dynamical system given by Eq. (3.1), $\mathbb{X}=(\phi, u, s)$. In the IS and NLCDH cases we also have the bulk pressure entering in the system as an additional function, $\mathbb{X}=(\phi, u, s, \Pi)$.

Writing $x=x_{0}+\delta x$, where we assume that $x_{0}$ is a stable solution of the system, which here will be taken as the slow-roll solutions that can be derived directly from Eq. (3.1) (see below), the equation for the variations in $\delta x$ become

$$
\delta \dot{\mathbb{X}}=\mathbb{M}\left(x_{0}\right) \delta \mathbb{X}-\dot{\mathbb{X}}_{0}
$$


where

$$
\mathbb{M}\left(x_{0}\right)=\frac{\partial \mathbb{F}\left(x_{0}\right)}{\partial x},
$$

is the Jacobian matrix for the system, evaluated at the $x_{0}$ solution, and $\dot{\mathbb{X}}_{0}$ is a residual force term, which in general is small and can be neglected [20]. The general solution of Eq. (3.5) is of the form

$$
\delta \mathbb{X}=\mathbb{X}_{0} e^{\mathbb{M}\left(x_{0}\right) t}
$$

and $\mathbb{M}\left(x_{0}\right)$ must be zero or negative for the system be stable, i.e., the eigenvalues $\Lambda_{i}$ of $\mathbb{M}\left(x_{0}\right)$ must necessarily all satisfy

$$
\Lambda_{i} \leq 0
$$

The stability of the dynamical system can be studied directly in the time variable, but it simplifies the analysis, in particular the determination of the eigenvalues of the Jacobian matrix, if we make a change of variables and rewrite the dynamical system (3.1) using $\phi$ as the independent variable instead of the time [20]. By doing this the dimension of the corresponding system is smaller and easier to analyze. In particular, the Jacobian matrix for the dynamical system (3.1) becomes a $2 \times 2$ matrix; if one includes the bulk pressure as an additional function to the system, as in IS and NLCDH cases, it then makes the Jacobian matrix $3 \times 3$. The eigenvalues obtained by using $\phi$ rather than time as the variable makes the analysis much simpler. As such, using that

$$
\frac{d}{d t}=\frac{d \phi}{d t} \frac{d}{d \phi}=u \frac{d}{d \phi}=u()^{\prime},
$$

where a prime indicates derivative with respect to $\phi$, the dynamical system Eq. (3.1) becomes equivalent to

$$
\begin{aligned}
u^{\prime} & =-3 H-\Upsilon-V_{, \phi} u^{-1}, \\
T s^{\prime} & =-3 H T s u^{-1}-3 H \Pi u^{-1}+\Upsilon u,
\end{aligned}
$$

together with the corresponding equations for the bulk viscosity, Eqs. (2.14), (2.20) or (2.27) (these last two also transformed to the $\phi$ variable), depending on which case we are considering.

The general solution of Eq. (3.10) is now of the form

$$
\delta \mathbb{X}=\mathbb{X}_{0} e^{\mathbb{M}\left(x_{0}\right) \phi(t)}
$$

The stability condition on the eigenvalues $\lambda_{i}$, which are the eigenvalues of $\mathbb{M}\left(x_{0}\right)$ once $u$ is factorized, depends now on whether the inflaton field $\phi(t)$ during slow-roll decreases with time (like in chaotic inflation), so $\lambda_{i} \geq 0$, or increases with time (like in hilltop inflation), in which case $\lambda_{i} \leq 0$. This is an important consideration when replacing the time by the inflaton as the independent variable in the dynamical system. In a $2 \times 2$ system, stability is ensured once the determinant is positive and the trace, negative, as is done in [20], [26]. However, in a $3 \times 3$ system, the case for the Israel-Stewart and NLCDH descriptions, further information beyond the trace and determinant is required to guarantee the stability of the system. That is why in this work we will make use directly of the eigenvalues for searching for the stability of the system. Nevertheless, for all the cases we have studied below, actually only one eigenvalue changes sign at the instability point, and therefore it would be enough to look at the determinant of the system.

As mentioned above, the stability analysis is done around the slow-roll solutions $x_{0}$. We study the stability of dynamical system around the slow-roll solutions, since they act as formal attractor solutions for the dynamical system [25, 38]. The slow-roll solutions are defined by the equations

$$
\begin{aligned}
u & =-\frac{V_{, \phi}}{3 H(1+Q)}, \\
T s & =Q u^{2}-\Pi, \\
\Pi & =-3 \zeta_{b} H,
\end{aligned}
$$


where we have omitted the index 0 that indicates the slow-roll solutions used in Eq. (3.5) for simplicity. Note that we are writing the slow-roll condition for $\Pi$ as the same in all three cases for the bulk viscous pressure that we have considered in this work. This is valid provided that the relaxation time $\tau$ is sufficiently small, which can be implicitly assumed by the condition (2.22). In Eq. (3.12) we defined $Q \equiv \Upsilon /(3 H)$, with the Hubble rate $H$, in the slow-roll approximation as given by

$$
H^{2}=\frac{1}{3 m_{\mathrm{P}}^{2}} V(1+\kappa),
$$

where $\kappa=\rho_{r} / V$. Keeping the radiation energy density in Eq. (3.13) is justified because in the presence of a bulk pressure, the radiation energy density does not in general need to be much smaller than the vacuum energy density in order to have inflation. In particular recall that in the presence of a bulk pressure, the acceleration equation is

$$
\frac{\ddot{a}}{a}=\frac{1}{6 m_{\mathrm{P}}^{2}}\left(2 V+3|\Pi|-2 \rho_{r}\right)
$$

which shows that we could in principle have $\rho_{r} \sim V$ and inflation could still be sustained by the bulk pressure [32].

The solutions (3.12) follow if the following conditions on slow-roll coefficients in warm inflation are satisfied [20],

$$
\begin{aligned}
\epsilon & =\frac{m_{\mathrm{P}}^{2}}{2}\left(\frac{V_{, \phi}}{V}\right)^{2} \ll 1+Q, \\
\eta & =m_{\mathrm{P}}^{2}\left(\frac{V_{, \phi \phi}}{V}\right) \ll 1+Q, \\
\beta & =m_{\mathrm{P}}^{2}\left(\frac{\Upsilon{ }_{, \phi} V_{, \phi}}{\Upsilon V}\right) \ll 1+Q, \\
b & =\frac{T V_{, T \phi}}{V_{, \phi}} \ll \frac{Q}{1+Q},
\end{aligned}
$$

where the slow-roll parameter $\beta$ takes into account the variation of $\Upsilon$ with respect to $\phi$, and the last condition for $b$ ensures that thermal corrections to the inflation potential are negligible.

Let us now study the stability of the dynamical system of equations in each of the three cases for the bulk pressure that we are considering.

\section{A. Dynamical system for the Eckart case}

In the Eckart theory the bulk pressure is simply given by Eq. (2.14), $\Pi=-3 \zeta_{b} H$. The dynamical system Eq. (3.10) takes the form,

$$
\begin{aligned}
u^{\prime} & =-3 H-\Upsilon-V_{, \phi} u^{-1} \equiv f(u, s), \\
s^{\prime} & =-3 H s u^{-1}+9 \zeta_{b} H^{2}(T u)^{-1}+\Upsilon T^{-1} u \equiv g(u, s) .
\end{aligned}
$$

The Jacobian matrix $\mathbb{M}$ becomes

$$
\mathbb{M}_{\text {Eckart }}(x)=\left.\left.\frac{\partial(f, g)}{\partial(u, s)}\right|_{u=u_{0}, s=s_{0}} \equiv\left(\begin{array}{ll}
\partial f / \partial u & \partial f / \partial s \\
\partial g / \partial u & \partial g / \partial s
\end{array}\right)\right|_{u=u_{0}, s=s_{0}}=\left(\begin{array}{ll}
\mathcal{A} & \mathcal{B} \\
\mathcal{C} & \mathcal{D}
\end{array}\right)
$$

where the matrix elements are evaluated at the slow-roll solutions Eqs. (3.12) and (3.13). The coefficients of the matrix $\mathbb{M}_{\text {Eckart }}$ become 


$$
\begin{aligned}
\mathcal{A} & =\frac{H}{u}\left\{-3(1+Q)-\frac{1}{(1+\kappa)^{2}} \frac{\epsilon}{(1+Q)^{2}}\right\}, \\
\mathcal{B} & =\frac{H}{s}\{-3(\gamma-1) c Q+3(\gamma-1) b(1+Q)+ \\
& \left.-\frac{1}{(1+\kappa)^{2}} \frac{Q \epsilon}{(1+Q)^{2}}+\frac{\sigma}{1+\kappa}\left[\frac{1}{(1+\kappa)} \frac{Q \epsilon}{(1+Q)^{2}}-\frac{3}{2} \tilde{\sigma}\right]\right\}, \\
\mathcal{C} & =\frac{H s}{u^{2}}\left[6-\frac{1}{(1+\kappa)^{2}} \frac{\epsilon}{(1+Q)^{2}}\right]\left\{1+\sigma \frac{\left[6(1+Q)^{2}-2 \epsilon\right]}{\left[6(1+Q)^{2}-\epsilon\right]}\right\}, \\
\mathcal{D} & =\frac{H}{u}\left\{3(\gamma-1)(c-1)-3-\frac{1}{(1+\kappa)^{2}} \frac{Q \epsilon}{(1+Q)^{2}}+\right. \\
& \left.+\sigma\left[3(\gamma-1)(c-l)-\frac{1}{(1+\kappa)^{2}} \frac{Q \epsilon}{(1+Q)^{2}}+\frac{3}{2} \frac{\tilde{\sigma}}{1+\kappa}\right]\right\},
\end{aligned}
$$

where we have omitted the sub-index "0" of the slow-roll solutions and defined the quantities $\sigma$ and $\tilde{\sigma}$ as

$$
\begin{aligned}
\sigma & =\frac{\Pi}{\gamma \rho_{r}}, \\
\tilde{\sigma} & =\frac{\Pi}{V} .
\end{aligned}
$$

The expressions simplify considerably in the strong dissipative regime of warm inflation, $Q \gg 1$ and neglecting the terms proportional to the slow-roll parameters in Eq. (3.18), we obtain

$$
\begin{aligned}
\mathcal{A} & =-3 Q \frac{H}{u}, \\
\mathcal{B} & =3(\gamma-1)(b-c) Q \frac{H}{s}, \\
\mathcal{C} & =\frac{H s}{u^{2}} 6(1+\sigma), \\
\mathcal{D} & =\frac{H}{u}\left\{3(\gamma-1)(c-1)-3+\left[3(\gamma-1)(c-l)+\frac{3}{2} \frac{\tilde{\sigma}}{1+\kappa}\right] \sigma\right\} .
\end{aligned}
$$

Using (3.21), the eigenvalues of $\mathbb{M}_{\text {Eckart }}$ are

$$
\begin{aligned}
\lambda_{1}^{\text {Eckart }} & \simeq-\frac{H}{u}[3 Q+6(1+\sigma)(\gamma-1)(b-c)]+\mathcal{O}(1 / Q), \\
\lambda_{2}^{\text {Eckart }} & \simeq \frac{H}{u}\left\{3(\gamma-1)(c-1)-3+\left[3(\gamma-1)(c-l)+\frac{3}{2} \frac{\tilde{\sigma}}{1+\kappa}\right] \sigma\right. \\
& +6(1+\sigma)(\gamma-1)(b-c)\}+\mathcal{O}(1 / Q) .
\end{aligned}
$$

Independent of the inflaton dynamics, we then obtain that stability is assured when $(u / H) \lambda_{i}<0$.

Note that in the slow-roll regime we have for $\sigma=\Pi /\left(\gamma \rho_{r}\right)$ that

$$
\sigma \simeq \frac{\Pi}{Q u^{2}-\Pi}=\frac{\tilde{\sigma}}{\frac{2 Q}{(1+\kappa)(1+Q)} \frac{\epsilon}{1+Q}-\tilde{\sigma}},
$$

where we have used the slow-roll equations for the radiation energy density and $u$, Eq. (3.12). Note from the above equation that in particular we have that $|\sigma|<1$. Using (3.24) in Eqs. (3.22) and (3.23), the first eigenvalue will always satisfy the stability condition, while for the second eigenvalue Eq. (3.23), the stability condition implies: 


$$
(c-2 b)(1+\sigma)+\frac{\gamma}{(\gamma-1)}+l \sigma-\frac{1}{2(\gamma-1)} \frac{\sigma \tilde{\sigma}}{1+\kappa}>0,
$$

or, using $\gamma=4 / 3$, valid for the quasi-equilibrium thermal bath of warm inflation,

$$
(c-2 b)(1+\sigma)+4+l \sigma-\frac{3}{2} \frac{\sigma \tilde{\sigma}}{1+\kappa}>0 .
$$

The Eq. (3.26) generalizes the results in [26], which were obtained for a constant bulk pressure $(l=0)$, by accounting for temperature dependence ${ }^{2}$. Also for $\sigma=0$ and $\tilde{\sigma}=0$, the case of zero bulk pressure, we reproduce the results obtained by Moss and Xiong in [20]. In [20] the stability condition was found to be $|c|<4$. From Eq. (3.26), in the absence of bulk viscosity, we derive instead only the condition $c>-4$. We do also obtain the result $c<4$ if we consider the eigenvalues in the approximation of very small dissipation $Q \ll 1$, but this regime is not the most general situation for warm inflation [39].

\section{B. Dynamical system for the IS case}

Let us now consider the dynamical system when the bulk pressure $\Pi$ has an evolution according to the IS theory, Eq. (2.20). The dynamical system, including the evolution equation for the bulk pressure, now becomes

$$
\begin{aligned}
u^{\prime} & =-3 H-\Upsilon-V_{, \phi} u^{-1} \equiv f(u, s, \Pi) \\
s^{\prime} & =-3 H s u^{-1}-3 H \Pi(T u)^{-1}+\Upsilon T^{-1} u \equiv g(u, s, \Pi) \\
\Pi^{\prime} & =-\frac{\Pi}{\tau} u^{-1}-\frac{3 \zeta_{b} H}{\tau} u^{-1}-\frac{\Pi}{2}\left\{3 H u^{-1}+\right. \\
& +\left[\frac{\tau_{, \phi}}{\tau}-\frac{\zeta_{b, \phi}}{\zeta_{b}}+(\gamma-1)\left(\frac{\tau, T}{\tau}-\frac{\zeta_{b, T}}{\zeta_{b}}-1\right) \frac{V_{, \phi T}}{s}\right]+ \\
& \left.-(\gamma-1)\left(\frac{\tau_{, T}}{\tau}-\frac{\zeta_{b, T}}{\zeta_{b}}-1\right)\left[3 H u^{-1}\left(1+\frac{\Pi}{T s}\right)-\frac{\Upsilon u}{T s}\right]\right\} \equiv h(u, s, \Pi),
\end{aligned}
$$

and the Jacobian stability matrix becomes

$$
\mathbb{M}_{I S}(x)=\left.\frac{\partial(f, g, h)}{\partial(u, s, \Pi)}\right|_{u=u_{0}, s=s_{0}, \Pi=\Pi_{0}}=\left(\begin{array}{ccc}
\mathcal{A} & \mathcal{B} & \mathcal{E} \\
\mathcal{C} & \mathcal{D} & \mathcal{F} \\
\mathcal{G} & \mathcal{H} & \mathcal{J}
\end{array}\right)
$$

Using the slow-roll solutions, Eqs. (3.12) and (3.13), we obtain for the elements of the matrix $\mathbb{M}_{I S}$ the result

\footnotetext{
${ }^{2}$ Our result given by Eq. (3.26) also corrects Eq. (22) in [26], where the authors of that reference mistakenly replaced $\tilde{\sigma}$ by $\sigma$.
} 


$$
\begin{aligned}
\mathcal{A} & =\frac{H}{u}\left[-3(1+Q)-\frac{1}{(1+\kappa)^{2}} \frac{\epsilon}{(1+Q)^{2}}\right] \\
\mathcal{B} & =\frac{H}{s}\{-3(\gamma-1) c Q+3(\gamma-1) b(1+Q)+ \\
& \left.-\frac{1}{(1+\kappa)^{2}} \frac{Q \epsilon}{(1+Q)^{2}}+\frac{\sigma}{1+\kappa}\left[\frac{1}{(1+\kappa)} \frac{Q \epsilon}{(1+Q)^{2}}-\frac{3}{2} \tilde{\sigma}\right]\right\}, \\
\mathcal{C} & =\frac{H s}{u^{2}}\left[6-\frac{1}{(1+\kappa)^{2}} \frac{\epsilon}{(1+Q)^{2}}\right](1+\sigma), \\
\mathcal{D} & =\frac{H}{u}\left[3(\gamma-1)(1+\sigma) c-3-3(\gamma-1)-\frac{1}{(1+\kappa)^{2}} \frac{Q \epsilon}{(1+Q)^{2}}\right], \\
\mathcal{E} & =0, \\
\mathcal{F} & =-3 \frac{H}{T u}, \\
\mathcal{G} & =\frac{H T s}{u^{2}} \sigma\left\{\frac{3}{2}+3(\gamma-1) \Lambda(1+\sigma)+\right. \\
& \left.+\frac{1}{3(1+\kappa)^{2}}\left[\frac{1}{\Theta}-\frac{3}{2}-\frac{3(\gamma-1)}{2} \Lambda(1+\sigma)\right] \frac{\epsilon}{(1+Q)^{2}}\right\} \\
\mathcal{H} & =\frac{H T}{u} \sigma\left\{\frac{(\gamma-1) l}{\Theta}+\frac{3(\gamma-1)^{2}}{2} c \Lambda(1+\sigma)-\frac{3 \gamma(\gamma-1)}{2} \Lambda+\right. \\
& +\frac{3(\gamma-1)}{2} b \frac{(1+Q)}{Q}(1+\sigma)\left[\frac{Q}{1+Q} \Lambda+(\gamma-1) \Sigma\right]+\frac{(\gamma-1)}{2(1+\kappa)} \frac{\chi}{1+Q}+ \\
& \left.+\frac{1}{3(1+\kappa)}\left[\frac{1}{\Theta}-\frac{3}{2}-3(\gamma-1) \Lambda(1+\sigma)\right]\left[\frac{1}{1+\kappa} \frac{Q \epsilon}{(1+Q)^{2}}-\frac{3}{2} \tilde{\sigma}\right]\right\} \\
\mathcal{J} & =\frac{H}{u}\left\{-\frac{1}{\Theta}-\frac{3}{2}-\frac{3(\gamma-1)}{2} \Lambda\left[\sigma+b \frac{(1+Q)}{Q}(1+\sigma)\right]+\frac{1}{2(1+\kappa)} \frac{\bar{g}-\bar{l}}{1+Q}\right\},
\end{aligned}
$$

where we have defined the parameters

$$
\begin{aligned}
& \Theta=\tau H, \quad \tilde{l}=\frac{T \zeta_{b, \phi T}}{\zeta_{b, \phi}}, \quad \bar{l}=\frac{1}{8 \pi G}\left(\frac{\zeta_{b, \phi} V_{, \phi}}{\zeta_{b} V}\right), \quad \hat{l}=\frac{T \zeta_{b, T T}}{\zeta_{b, T}}, \\
& g=\frac{T \tau_{, T}}{\tau}, \quad \tilde{g}=\frac{T \tau_{, \phi T}}{\tau_{, \phi}}, \quad \bar{g}=\frac{1}{8 \pi G}\left(\frac{\tau_{, \phi} V_{, \phi}}{\tau V}\right), \quad \hat{g}=\frac{T \tau_{, T T}}{\tau_{, T}}, \\
& \Lambda=1+l-g, \quad \chi=(\tilde{g}-g) \bar{g}-(\tilde{l}-l) \bar{l}, \\
& \Sigma=(1+\hat{g}-g) g-(1+\hat{l}-l) l-\Lambda \hat{b}, \quad \hat{b}=T V_{, \phi T T} / V_{, \phi T} .
\end{aligned}
$$

In the strong dissipation regime $Q \gg 1$ and neglecting terms proportional to the slow-roll coefficients, Eq. (3.29) simplifies considerably and we obtain 


$$
\begin{aligned}
\mathcal{A} & =-3 Q \frac{H}{u}, \\
\mathcal{B} & =3(\gamma-1)(b-c) Q \frac{H}{s}, \\
\mathcal{C} & =\frac{H s}{u^{2}} 6(1+\sigma), \\
\mathcal{D} & =\frac{H}{u}[3(\gamma-1)(1+\sigma) c-3-3(\gamma-1)], \\
\mathcal{E} & =0, \\
\mathcal{F} & =-3 \frac{H}{T u} \\
\mathcal{G} & =\frac{H T s}{u^{2}} \sigma\left[\frac{3}{2}+3(\gamma-1) \Lambda(1+\sigma)\right], \\
\mathcal{H} & =\frac{H T}{u} \sigma\left\{\frac{(\gamma-1) l}{\Theta}+\frac{3(\gamma-1)^{2}}{2} c \Lambda(1+\sigma)-\frac{3 \gamma(\gamma-1)}{2} \Lambda+\right. \\
& +\frac{3(\gamma-1)}{2} b(1+\sigma)[\Lambda+(\gamma-1) \Sigma]+ \\
& \left.-\frac{\tilde{\sigma}}{2(1+\kappa)}\left[\frac{1}{\Theta}-\frac{3}{2}-3(\gamma-1) \Lambda(1+\sigma)\right]\right\}, \\
\mathcal{J} & =\frac{H}{u}\left\{-\frac{1}{\Theta}-\frac{3}{2}-\frac{3(\gamma-1)}{2} \Lambda[\sigma+b(1+\sigma)]\right\} .
\end{aligned}
$$

In terms of Eq. (3.32), the eigenvalues of $\mathbb{M}_{I S}$ are

$$
\begin{aligned}
& \lambda_{1}^{\mathrm{IS}} \simeq-\frac{H}{u}[3 Q+6(1+\sigma)(\gamma-1)(b-c)]+\mathcal{O}(1 / Q), \\
& \lambda_{2}^{\mathrm{IS}} \simeq \frac{1}{2}\left(\mathcal{D}-\frac{\mathcal{B C}}{\mathcal{A}}+\mathcal{J}\right)+\frac{1}{2}\left[(\mathcal{D}-\mathcal{J})\left(\mathcal{D}-\mathcal{J}-2 \frac{\mathcal{B C}}{\mathcal{A}}\right)-4 \mathcal{F}\left(\frac{\mathcal{B} \mathcal{G}}{\mathcal{A}}-\mathcal{H}\right)+\frac{\mathcal{B}^{2} \mathcal{C}^{2}}{\mathcal{A}^{2}}\right]^{1 / 2}+\mathcal{O}(1 / Q), \\
& \lambda_{3}^{\mathrm{IS}} \simeq \frac{1}{2}\left(\mathcal{D}-\frac{\mathcal{B C}}{\mathcal{A}}+\mathcal{J}\right)-\frac{1}{2}\left[(\mathcal{D}-\mathcal{J})\left(\mathcal{D}-\mathcal{J}-2 \frac{\mathcal{B C}}{\mathcal{A}}\right)-4 \mathcal{F}\left(\frac{\mathcal{B} \mathcal{G}}{\mathcal{A}}-\mathcal{H}\right)+\frac{\mathcal{B}^{2} \mathcal{C}^{2}}{\mathcal{A}^{2}}\right]^{1 / 2}+\mathcal{O}(1 / Q) .
\end{aligned}
$$

For the first eigenvalue above, $\lambda_{1}^{\mathrm{IS}}$, we see that it is the same as the Eckart case, $\lambda_{1}^{\mathrm{Eckart}}$, so it already satisfies the stability requirement that $(u / H) \lambda_{i}<0$. Adding all the factors in the two other eigenvalues in Eqs. (3.34) and (3.35), using that $\Theta \ll 1$, we obtain that

$$
\lambda_{2}^{\mathrm{IS}} \simeq-\frac{H}{u}\left\{\frac{1}{\Theta}+3|\sigma|\left[(\gamma-1) l+\frac{|\tilde{\sigma}|}{2(1+\kappa)}\right]\right\}+\mathcal{O}(\Theta)
$$

and it also satisfies the stability requirement. The stability condition for the IS case then falls on the third eigenvalue $\lambda_{3}^{\mathrm{IS}}$, Eq. (3.35). This is most easily expressed by demanding that the product $\lambda_{2}^{\mathrm{IS}} \lambda_{3}^{\mathrm{IS}}>0$, which then leads to the condition

$$
\begin{aligned}
& {\left[1+\frac{2 \sigma+3(\gamma-1) \Theta b \Lambda(1+\sigma)^{2}}{2+3 \Theta}\right] c+\frac{\gamma}{\gamma-1}+\frac{2 \sigma}{2+3 \Theta} l} \\
& -\frac{1}{2(\gamma-1)} \frac{\sigma \tilde{\sigma}}{1+\kappa}\left[\frac{2-3 \Theta}{2+3 \Theta}-\frac{6(\gamma-1) \Theta \Lambda}{2+3 \Theta}(1+\sigma)\right]+ \\
& -\left[4(1+\sigma)+3 \Theta(2+\sigma)-3 \Theta \Lambda(1+\sigma)^{2}+\right. \\
& \left.-3(\gamma-1) \Theta(1+\sigma)(\Lambda+\sigma \Sigma)+6 b(\gamma-1) \Lambda \Theta(1+\sigma)^{2}\right] \frac{b}{2+3 \Theta}>0
\end{aligned}
$$

which for $\gamma=4 / 3$ becomes 


$$
\begin{aligned}
& {\left[1+\frac{2 \sigma+\Theta b \Lambda(1+\sigma)^{2}}{2+3 \Theta}\right] c+4+\frac{2 \sigma}{2+3 \Theta} l-\frac{3}{2} \frac{\sigma \tilde{\sigma}}{1+\kappa}\left[\frac{2-3 \Theta}{2+3 \Theta}-\frac{2 \Theta \Lambda}{2+3 \Theta}(1+\sigma)\right]+} \\
& -\left[4(1+\sigma)+3 \Theta(2+\sigma)-3 \Theta \Lambda(1+\sigma)^{2}-\Theta(1+\sigma)(\Lambda+\sigma \Sigma)+2 b \Lambda \Theta(1+\sigma)^{2}\right] \frac{b}{2+3 \Theta}>0 .
\end{aligned}
$$

From Eq. (3.38), when the relaxation time vanishes, $\tau=0$, i.e., for $\Theta=\tau H \rightarrow 0$, we recover the previous condition Eq. (3.26), obtained in the Eckart theory case.

\section{Dynamical system for the NLCDH case}

Finally, we will now obtain stability condition for the case of the NLCDH theory for the bulk pressure. In the

$\mathrm{NLCDH}$ case, the evolution equation for the bulk pressure is given by Eq. 2.27). The dynamical system now becomes

$$
\begin{aligned}
u^{\prime} & =-3 H-\Upsilon-V_{, \phi} u^{-1} \equiv f(u, s, \Pi), \\
s^{\prime} & =-3 H s u^{-1}-3 H \Pi(T u)^{-1}+\Upsilon T^{-1} u \equiv g(u, s, \Pi), \\
\Pi^{\prime} & =-\frac{\Pi}{\tau} u^{-1}-\frac{3 \zeta_{b} H}{\tau} u^{-1}-3 H \Pi u^{-1} \equiv h(u, s, \Pi) .
\end{aligned}
$$

The Jacobian stability matrix is similar to the one in the IS case, Eq. (3.28), but now with the functions $f(u, s, \Pi), g(u, s, \Pi), h(u, s, \Pi)$ obtained from the above equation (3.39). Using again the slow-roll solutions, Eqs. (3.12) and (3.13), we obtain for the elements of the matrix $\mathbb{M}_{N L C D H}$ in the NLCDH case,

$$
\begin{aligned}
\mathcal{A} & =\frac{H}{u}\left\{-3(1+Q)-\frac{1}{(1+\kappa)^{2}} \frac{\epsilon}{(1+Q)^{2}}\right\} \\
\mathcal{B} & =\frac{H}{s}\{-3(\gamma-1) c Q+3(\gamma-1) b(1+Q)+ \\
& \left.-\frac{1}{(1+\kappa)^{2}} \frac{Q \epsilon}{(1+Q)^{2}}+\frac{\sigma}{1+\kappa}\left[\frac{1}{(1+\kappa)} \frac{Q \epsilon}{(1+Q)^{2}}-\frac{3}{2} \tilde{\sigma}\right]\right\}, \\
\mathcal{C} & =\frac{H s}{u^{2}}\left[6-\frac{1}{(1+\kappa)^{2}} \frac{\epsilon}{(1+Q)^{2}}\right](1+\sigma), \\
\mathcal{D} & =\frac{H}{u}\left\{3(\gamma-1)(1+\sigma) c-3-3(\gamma-1)-\frac{1}{(1+\kappa)^{2}} \frac{Q \epsilon}{(1+Q)^{2}}\right\}, \\
\mathcal{E} & =0 \\
\mathcal{F} & =-3 \frac{H}{T u}, \\
\mathcal{G} & =\frac{H T s}{u^{2}} \sigma\left[3+\frac{1}{3(1+\kappa)^{2}}\left(\frac{1}{\Theta}-\frac{3}{2}\right) \frac{\epsilon}{(1+Q)^{2}}\right], \\
\mathcal{H} & =\frac{H T}{u} \sigma\left\{\frac{(\gamma-1) l}{\Theta}+\frac{1}{3(1+\kappa)}\left(\frac{1}{\Theta}-\frac{3}{2}\right)\left[\frac{1}{(1+\kappa)} \frac{Q \epsilon}{(1+Q)^{2}}-\frac{3}{2} \tilde{\sigma}\right]\right\}, \\
\mathcal{J} & =\frac{H}{u}\left(-\frac{1}{\Theta}-3\right),
\end{aligned}
$$

with parameters the same as defined in Eq. (3.30).

Taking again the strong dissipative regime, $Q \gg 1$, and neglecting terms proportional to the slow-roll coefficients, Eq. (3.40) simplifies to 


$$
\begin{aligned}
\mathcal{A} & =-3 Q \frac{H}{u}, \\
\mathcal{B} & =3(\gamma-1)(b-c) Q \frac{H}{s}, \\
\mathcal{C} & =\frac{H s}{u^{2}} 6(1+\sigma), \\
\mathcal{D} & =\frac{H}{u}[3(\gamma-1)(1+\sigma) c-3-3(\gamma-1)], \\
\mathcal{E} & =0 \\
\mathcal{F} & =-3 \frac{H}{T u}, \\
\mathcal{G} & =\frac{H T s}{u^{2}} 3 \sigma, \\
\mathcal{H} & =\frac{H T}{u} \sigma\left[\frac{(\gamma-1) l}{\Theta}-\frac{1}{2}\left(\frac{1}{\Theta}-3\right) \frac{\tilde{\sigma}}{1+\kappa}\right], \\
\mathcal{J} & =\frac{H}{u}\left(-\frac{1}{\Theta}-3\right) .
\end{aligned}
$$

The eigenvalues that follow from $\mathbb{M}_{N L C D H}$, using (3.41) and considering the high dissipative regime $Q \gg 1$, are still of the form obtained in the IS case, Eqs. (3.33), (3.34) and (3.35), with coefficients as given by (3.41). Obviously, one of the eigenvalue is still the same as the one obtained in the Eckart case, Eq. (3.22), while the other two determine the stability condition for the NLCDH case, similar to Eq. (3.37),

$$
\left(1+\frac{\sigma}{1+3 \Theta}\right) c+\frac{\gamma}{\gamma-1}+\frac{\sigma}{1+3 \Theta} l-\frac{1}{2(\gamma-1)} \frac{\sigma \tilde{\sigma}}{1+\kappa} \frac{1-3 \Theta}{1+3 \Theta}-\left[2+\sigma+\frac{\sigma}{1+3 \Theta}\right] b>0 .
$$

For $\gamma=4 / 3$ the above equation gives

$$
\left(1+\frac{\sigma}{1+3 \Theta}\right) c+4+\frac{\sigma}{1+3 \Theta} l-\frac{3}{2} \frac{\sigma \tilde{\sigma}}{1+\kappa} \frac{1-3 \Theta}{1+3 \Theta}-\left(2+\sigma+\frac{\sigma}{1+3 \Theta}\right) b>0 .
$$

If we take the relaxation time as vanishing in Eq. (3.43), $\Theta=\tau H \rightarrow 0$, we once again recover the result Eq. (3.26).

\section{NUMERICAL RESULTS}

In this Section, we study numerically the system of equations for each of the three cases derived in the previous section. We will verify the corresponding stability conditions directly through the numerical time evolution of the corresponding dynamical systems. We will restrict to models of warm inflation that can be derived from quantum field theory. Moreover to develop the basic ideas, we will restrict to a dissipation coefficient of the form [8, 13, 15] $\Upsilon=C_{\phi} T^{3} / \phi^{2}$, which is what is obtained from the two-stage mechanism in the low temperature regime. Although our analysis in this paper is restricted to this particular dissipative coefficient, it can easily be extended to other dissipative coefficients, with various other types studied in [7, 13 -15]. The bulk viscosity coefficient will have the form $\zeta_{b}=C_{b} T^{3}$, which is obtained for quantum fields in the high temperature regime 24] and also is the form generically considered in hydrodynamics.. This then corresponds to the case where $c=3$ for the dissipative coefficient in Eq. (3.3) and $l=3$ for the bulk viscosity, with $C_{\phi}$ and $C_{b}$ (dimensionless) proportionality constants. Also for simplicity, we will analyze here the simplest case of a quadratic inflaton potential,

$$
V=\frac{m_{\phi}^{2}}{2} \phi^{2},
$$

with a constant relaxation time $(g=\hat{g}=\tilde{g}=\bar{g}=0$ in the constants defined in (3.30) $)$. The extension to other types of potentials, such as a quartic potential or hybrid type potentials, does not offer additional difficulties and can be easily implemented. 
In the example considered here, the stability conditions Eqs. (3.26), (3.38) and (3.43), for the Eckart, IS and NLCDH cases respectively, reduce to

$$
\begin{aligned}
C_{\text {stab }}^{\text {Eckart }} & =3(1+\sigma)+4+3 \sigma-\frac{3}{2} \frac{\sigma \tilde{\sigma}}{1+\kappa}>0, \\
C_{\text {stab }}^{I S} & =3\left(1+\frac{2}{2+3 \Theta} \sigma\right)+4+\frac{6 \sigma}{2+3 \Theta}+ \\
& -\frac{3}{2} \frac{\sigma \tilde{\sigma}}{1+\kappa}\left[\frac{2-3 \Theta}{2+3 \Theta}-\frac{8 \Theta}{2+3 \Theta}(1+\sigma)\right]>0, \\
C_{\text {stab }}^{N L C D H} & =3\left(1+\frac{\sigma}{1+3 \Theta}\right)+4+\frac{3 \sigma}{1+3 \Theta}-\frac{3}{2} \frac{\sigma \tilde{\sigma}}{1+\kappa} \frac{1-3 \Theta}{1+3 \Theta}>0 .
\end{aligned}
$$

In all the numerical studies using the inflaton potential Eq. (4.1), we have kept fixed the values $m_{\phi}=\sqrt{8 \pi} \times 10^{-6} m_{\mathrm{P}}$, the initial value for the dissipation factor $Q=100$, the initial temperature $T=370 m_{\phi}$ and $\phi(0)=10.98 m_{\mathrm{P}}$. The values for $H(0)$ and $\dot{\phi}(0)$ follow from the slow-roll conditions. These values correspond to a proportionality constant $C_{\phi} \simeq 1.61 \times 10^{8}$ for the dissipation term, which is a typical value found in the context of WI model building [40]. The value of the bulk viscosity coefficient $C_{b}$ is varied and also the value of the relaxation constant $\tau H=\Theta$, but observing that we are still in the region of validity of the thermal radiation bath at quasi-equilibrium, $\Theta<1$.

By letting the system of equations evolve, we determine the critical value of $C_{b}$ for which the stability conditions for each of the three theories studied here, Eqs. (4.2), (4.3) and (4.4), are violated. The corresponding results are given in Tab. []

\begin{tabular}{c|c|c}
\hline$\Theta$ & theory & $C_{b}$ \\
\hline & Eckart & 2232.94 \\
0.01 & IS & 2266.48 \\
& NCLDH & 2300.04 \\
\hline & Eckart & 2232.94 \\
0.05 & IS & 2400.61 \\
& NCLDH & 2568.42 \\
\hline & Eckart & 2232.94 \\
0.1 & IS & 2568.23 \\
& NCLDH & 2903.79 \\
\hline
\end{tabular}

TABLE I: The critical values for the bulk viscosity constant $C_{b}$. The Eckart case is independent of $\Theta$, therefore the value for its critical $C_{b}$ does not change.

We note from the results of Tab. \that the values for the bulk viscosity constant $C_{b}$ for which the stability conditions for IS and NCLDH cases are violated increases with respect to the Eckart case as $\Theta$ increases. In Tab. II we give the corresponding differences in percentage.

\begin{tabular}{c|c|c|c}
\hline theory & $\Theta=0.01$ & $\Theta=0.05$ & $\Theta=0.10$ \\
\hline IS & $1.5 \%$ & $7.5 \%$ & $15.0 \%$ \\
\hline NLCDH & $3.0 \%$ & $15.0 \%$ & $30.0 \%$ \\
\hline
\end{tabular}

TABLE II: The increase of the critical value of $C_{b}$ for the causal theories with respect to the Eckart theory.

Once we have the system evolving in time and also allowing the time dependence for the stability parameters $C_{\text {stab }}^{\text {Eckart }}, C_{\text {stab }}^{\mathrm{IS}}$ and $C_{\text {stab }}^{\mathrm{NLCDH}}$, by starting from the initial conditions given above, we can explicitly check that the time where Eqs. (4.2), (4.3) and (4.4), are violated, is the time where both radiation energy density and the bulk pressure start to grow exponentially, as expected. In the Figs. 1, 2 and 3 we plot the stability parameters $C_{\text {stab }}^{\text {Eckart }}, C_{\text {stab }}^{\mathrm{IS}}$ and $C_{\mathrm{stab}}^{\mathrm{NLCH}}$ along side those for the radiation energy density and the bulk pressure, as a function of time, for the case of $\Theta=0.01$ and for the values of critical $C_{b}$ shown in Tab. []

The results shown in Figs. 1, 2 and 3 indicate that the time where the stability condition is violated corresponds to an inflection point in the radiation energy density and the bulk pressure evolutions. After that time, both the radiation energy density and the bulk pressure start to grow and soon after the dynamics become uncontrollable. The point where the stability conditions Eqs. (4.2), (4.3) and (4.4), are violated, corresponds then to a turnover point in the evolution of the dynamical system of equations. 

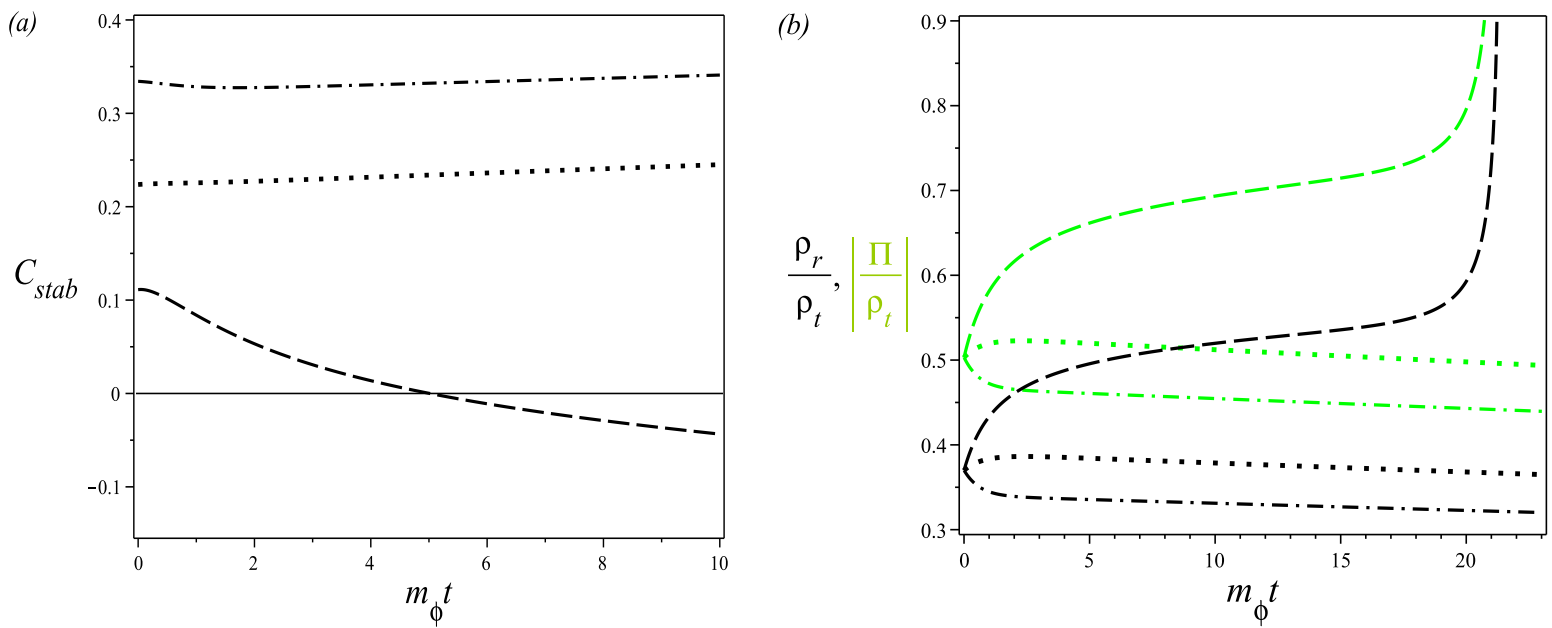

FIG. 1: The stability condition (a) and the results (b) for the radiation energy density, $\rho_{r}$ (black curves), and bulk pressure, $\Pi$ (green curves), normalized by the total energy density. The dashed curves are for the Eckart case, the dotted curves are for IS and the dash-dotted curves are for NLCDH. In all cases $\Theta=0.01$ and $C_{b}=2232.94$.
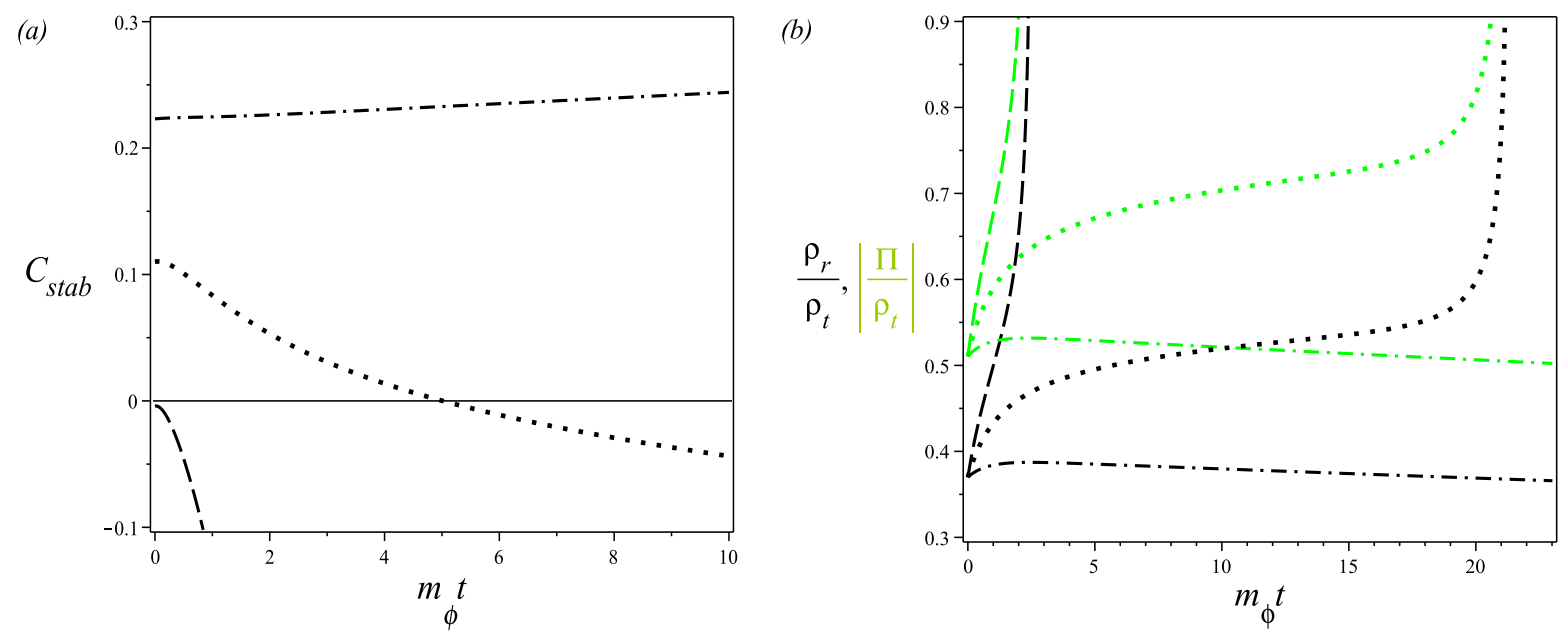

FIG. 2: The same as in Fig. 1, but now for the critical value $C_{b}=2266.48$.

We can also notice from the results for the radiation and bulk pressure shown in Figs. 10, $2 \mathrm{~b}$ and $3 \mathrm{~b}$, that the causal theories always lead to a smaller radiation production when compared to the noncausal case. Among the two causal theories studied here, the NLCDH theory of Ref. 31] gives a much smaller radiation production from bulk pressure effects than the IS theory. The differences between the causal theories of IS and NLCDH are also larger than the noncausal theory of Eckart as the relaxation time increases, which is clear from the results presented in Tabs. I] and III. Even though the difference of the Eckart theory for the bulk pressure from that of IS is around the percent level for a relatively small relaxation time of $\tau H=0.01$, it is twice that (in percentage) when the NLCDH theory is considered. This difference between the IS and NLCDH theories can easily be understood once we compare the Eqs. (2.20) and (2.27) and realizes that the relaxation time in the NLCDH theory appears with an additional factor two on the right-hand-side of the equation. Among the theories for the bulk pressure we have studied here, thus, the NLCDH case is the most robust in terms of stability. It allows for relatively larger bulk viscous pressures as compared to the Eckart and IS cases. 

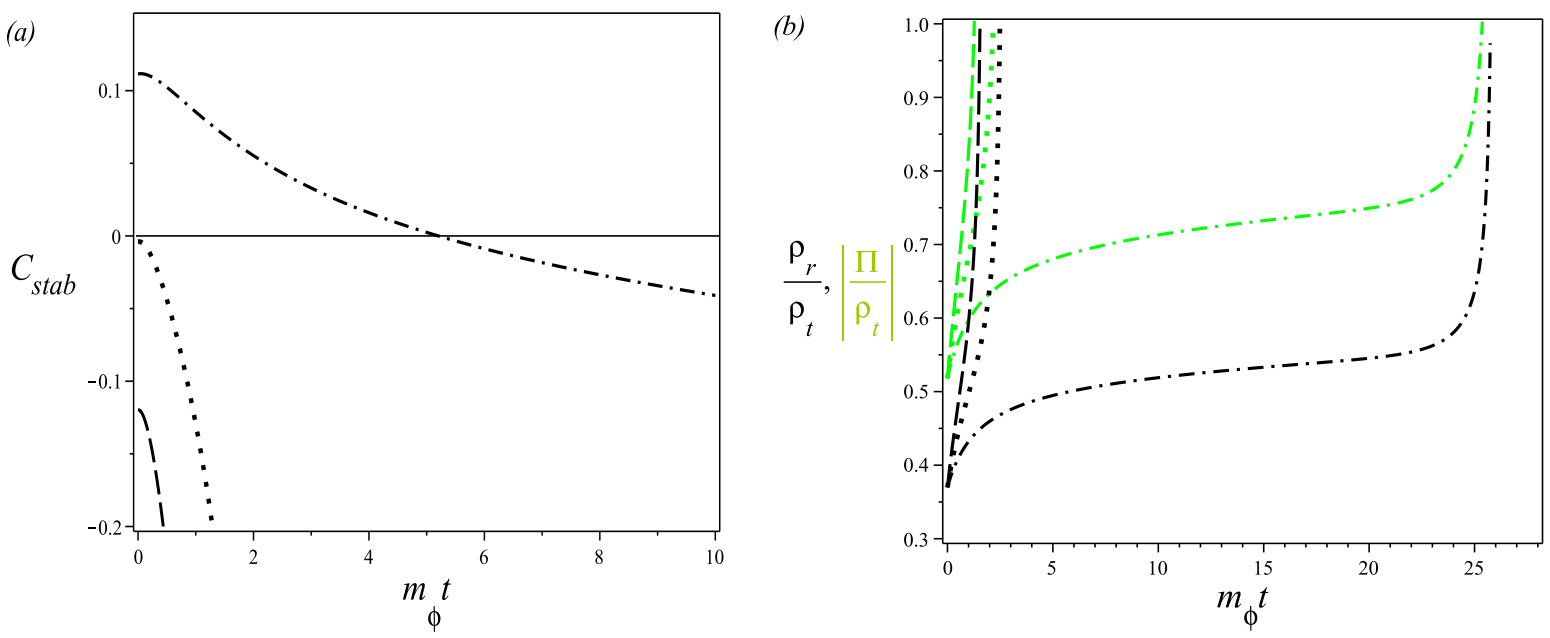

FIG. 3: The same as in Fig. 1] but now for the critical value $C_{b}=2300.04$.

\section{BULK VISCOSITY COEFFICIENTS FROM QUANTUM FIELD THEORY}

The shear and bulk viscosities describe the properties of a system to return to equilibrium when displaced from it. As explained in 24], at the level of particle physics processes, these viscosities are generally proportional to the mean free path, or equivalently time, of the relevant scattering process. The bulk viscosity is proportional to the mean free path for particle number changing processes in theories with breakdown of scale invariance. In contrast the shear viscosity is proportional to the two-body elastic scattering mean free path. Thus the bulk viscosity roughly has the form

$$
\zeta_{b} \sim \tilde{m}^{4} \tau
$$

where $\tilde{m}$ is a characteristic measure of the violation from scale invariance in the theory and $\tau$ is the mean free time between number changing inelastic scattering processes.

Explicit expressions for the bulk viscosity have been calculated for a self-interacting $\lambda_{y} y^{4}$ scalar field theory model in 24] for different temperature regimes. The obtained results for the bulk viscosity relevant for us here are 24],

$$
\zeta_{b} \simeq\left\{\begin{array}{l}
5.5 \times 10^{4} \frac{\tilde{m}_{y}^{4} m_{y}^{2}(T)}{\lambda_{y}^{4} T^{3}} \ln ^{2}\left[1.2465 m_{y}(T) / T\right], m_{y} \ll T \ll m_{y} / \lambda_{y} \\
8.9 \times 10^{-5} \lambda_{y} T^{3} \ln ^{2}\left(0.064736 \lambda_{y}\right), \quad T \gg m_{y} / \lambda_{y},
\end{array}\right.
$$

where $m_{y}(T)$ is the scalar $y$ field thermal mass, $m_{y}^{2}(T)=m_{y}^{2}+\lambda_{y} T^{2} / 24\left[1+\mathcal{O}\left(m_{y} / T\right)\right]$, and $\tilde{m}_{y}^{2} \equiv m_{y}^{2}(T)-$ $T^{2}\left(\partial m_{y}^{2}(T) / \partial T^{2}\right) \simeq m_{y}^{2}-\beta\left(\lambda_{y}\right) T^{2} / 48$, where $\beta\left(\lambda_{y}\right)=3 \lambda_{y}^{2} /\left(16 \pi^{2}\right)$ is the renormalization group $\beta$-function. Note that even for a massless scalar field at tree-level, $m_{y}=0$ and thus it is classically scale invariant, the scale invariance is broken quantum mechanically. In this case, the $\beta$-function gives a measure of breaking of scale invariance.

The characteristic relaxation time relevant for the bulk viscosity and that which also enters in the IS and NLCDH formulas for the bulk pressure, can be extracted from the result for the bulk viscosity given by Eq. (5.2) and the formal expression for it in the context of the Kubo formula for $m_{y} \ll T$ and in the relaxation time approximation [41],

$$
\zeta_{b}=\frac{1}{T} \int \frac{d^{3} p}{(2 \pi)^{3}} \frac{\tau\left(\omega_{p}\right)}{\omega_{p}^{2}} n\left(\omega_{p}\right)\left[1+n\left(\omega_{p}\right)\right]\left[\left(\frac{1}{3}-v_{s}^{2}\right) \mathbf{p}^{2}-v_{s}^{2} \tilde{m}_{y}^{2}\right]^{2}
$$

where $n\left(\omega_{p}\right)=1 /\left[\exp \left(\omega_{p} / T\right)-1\right]$ is the Bose-Einstein distribution, $\omega_{p}=\sqrt{\mathbf{p}^{2}+m_{y}^{2}(T)}$ and $v_{s}$ is the speed of sound for the radiation bath of scalar $y$-particles. Using an on-shell approximation for the relaxation time, $\tau\left(\omega_{p}\right) \simeq \tau \omega_{p} / m_{y}(T)$, where $\tau \equiv \tau\left(m_{y}(T)\right)=$ constant, and the result for the speed of sound for a self-interacting scalar field in the hightemperature approximation $m_{y} \ll T$ [24], $v_{s}^{2} \simeq 1 / 3-5 \tilde{m}_{y}^{2} /\left(12 \pi^{2} T^{2}\right)$, we obtain for the ratio $\zeta_{b} / \tau$ the result: 


$$
\begin{aligned}
\frac{\zeta_{b}}{\tau} & \simeq \tilde{m}_{y}^{4} \frac{1}{m_{y}(T) T} \int \frac{d^{3} p}{(2 \pi)^{3}} \frac{1}{\omega_{p}} n\left(\omega_{p}\right)\left[1+n\left(\omega_{p}\right)\right]\left(\frac{5 \mathbf{p}^{2}}{12 \pi^{2} T^{2}}-\frac{1}{3}\right)^{2} \\
& \simeq \frac{\tilde{m}_{y}^{4} T}{18 \pi^{2} m_{y}(T)} \ln \left(\frac{2 T}{m_{y}(T)}\right) .
\end{aligned}
$$

Note that for the above results for the bulk viscosity to be applicable in warm inflation, it is required that the effective mass for the scalar $y$ field be larger than the Hubble parameter, $m_{y}(T) \gg H$. In this case curvature corrections to the quantum field expressions defining the bulk viscosity can be neglected and the Minkowski expression (5.2) applies. Also, as already pointed out in the previous sections, a quasi-equilibrium thermal radiation bath requires that the relevant relaxation time, set by $\tau$ be also short compared to the Hubble time, $\tau<1 / H$. These conditions can be easily meet for warm inflation as verified in the next section.

\section{MODEL BUILDING}

We have understood how to separate, in general, between the stable and unstable regimes. Moreover, we have studied the differences between the non-causal and causal descriptions of the bulk viscosity. In this Section, we are going to apply this knowledge to some generic supersymmetric (SUSY) models of warm inflation, namely, the chaotic, hybrid and hilltop (or new inflation) models. As an aside, recall that for single field, cold inflation models, cosmic microwave background radiation (CMB) data 42] is currently able to exclude many of the chaotic type of models, with potential $V \sim \phi^{n}$, such as with polynomial power $n=3$ (at 95\% CL) and higher powers $(n>3$ ). Also typical hybrid inflation models are disfavored by the CMB data for having too large a spectral index $n_{s}>1$, while new inflation (hilltop) type cold inflation models seem to be more favored by the data 43]. However, these conclusions do not apply to warm inflation models [40], and in particular to large field inflation models with dissipation [26, 39], for which the predictions related to the primordial spectrum (spectral index, running of the spectral index, non-gaussianity parameter) are different from those of standard cold inflation. In this paper we have only focused on the background evolution and so these observational details are not directly relevant. A detailed study of the primordial spectrum, that includes dissipation and viscous effects and the observational consequences, will be presented elsewhere. The goal of this Section is to study the parameter space of these basic inflation models in the presence of bulk viscosity.

As discussed in previous sections, the bulk viscous pressure decreases the radiation pressure and so allows the source term creating radiation to be more effective. Thus assuming thermalization, it raises the temperature. Given that the dissipative coefficient depends on the temperature, the bulk viscosity enhances it; and therefore, the inflaton can slow-roll down its potential with lower values of the dissipative factor $C_{\phi}$. Hence, we expect an enlargement of the parameter space in regions of low $C_{\phi}$, where warm inflation is not allowed in the absence of bulk viscosity. In addition, in the regions of the parameter space where warm inflation is allowed without bulk viscosity, its effect is to produce more e-folds of inflation.

One of the main issues that need to be faced in warm inflation are the quantum and thermal corrections to the inflaton potential, that tend to spoil the required flatness of the potential needed for inflation. We invoke SUSY to avoid the quantum corrections, which at the same time does not cancel the time non-local terms that give rise to the dissipative terms. The pattern of interactions leading to warm inflation is given by the superpotential:

$$
W=W(\Phi)+g \Phi X^{2}+h X Y^{2},
$$

where $\Phi, X$ and $Y$ denote superfields. The regime most studied for warm inflation is where the $X$ field, being coupled to the inflaton field, is massive during inflation, and therefore thermal corrections to the inflaton mass are Boltzmann suppressed as long as $m_{\chi}>T$, where $\chi$ is the scalar component of the superfield $X$ and $m_{\chi}^{2}=2 g^{2} \phi^{2}$. Even though the $X$ field in (6.1) is at low temperatures, we can still have the $Y$ field at high temperatures, e.g., $m_{y} \ll T$, where $y$ is the scalar component of $Y$ and $m_{y}$ its mass. In this case, thermal dissipation of the inflaton field mediated by $X$ decaying into $Y$ is not Boltzmann suppressed [13] and can lead to a warm inflation regime. We denote this regime as the low- $T$ regime and it leads to a dissipative coefficient of the form [8, 13, 15] $\Upsilon=C_{\phi} T^{3} / \phi^{2}$. Other regimes and dissipative coefficients could also be studied, but we will develop the basic methodology in this paper with this example.

Warm inflation imposes another condition on the temperature, $T>H$. This condition roughly separates warm inflation from cold inflation, since for $T<H$ dissipation effects are negligible. We also must take into account the thermodynamical condition $\left|\Pi / p_{r}\right|<1$, as the hydrodynamic descriptions of the bulk viscosity that we are using treat the viscous pressure as a perturbation to the equilibrium one. 
Therefore, we define the available parameter space for warm inflation as the regions where the following conditions hold:

1. $\epsilon_{H}=-\dot{H} / H^{2}<1$, which is the standard condition for the accelerated expansion,

2. $\rho_{\phi}>\rho_{r}$, which prevents the radiation energy density to dominate,

3. $T / H>1$, which leads to non-negligible dissipation effects,

4. $\phi / T \gtrsim 10$, which is the low- $T$ condition for $g=\mathcal{O}(1)$,

5. $\left|\Pi / p_{r}\right|<1$, which is the condition for the hydrodynamic description to hold.

These conditions need to hold for at least 40 e-folds to solve the flatness and horizon problems. In standard cold inflation, this value is taken to be 50-60 e-folds; nevertheless, the exact number depends on the scale of inflation and the initial post-inflation temperature. As warm inflation usually decreases both quantities, it is very reasonable to demand 40 e-folds. Condition 2 is controlled by the stability conditions found in the previous section. From system of equations (3.12), we can relate the rest of the conditions with slow-roll parameters (3.15). Condition 1 during slow-roll simply is:

$$
\epsilon_{H}=\frac{\epsilon}{1+Q}<1
$$

The evolution of the ratio $T / H$, in the slow-roll regime, with respect to the number of e-folds is given by:

$$
\frac{d \ln (T / H)}{d N_{e}}=\frac{2(1+\sigma)}{1+Q+6 Q(1+\sigma)}\left(\frac{2+4 Q}{1+Q} \epsilon-\eta+\frac{1-Q}{1+Q} \frac{m_{P}}{\phi} \sqrt{2 \epsilon}\right) .
$$

$\Pi / p_{r}$ is directly related to $T / H$,

$$
\left|\frac{\Pi}{p_{r}}\right|=\frac{270 C_{b}}{\pi^{2} g_{*}} \frac{H}{T}
$$

The evolution of $\phi / T$ by:

$$
\frac{d \ln (\phi / T)}{d N_{e}}=\frac{-1}{[1+Q+6 Q(1+\sigma)]}\left[\frac{3+4 \sigma+(1+2 \sigma) Q}{1+Q} \epsilon-2(1+\sigma) \eta+\frac{3+2 \sigma+(5+4 \sigma) Q}{1+Q} \frac{m_{P}}{\phi} \sqrt{2 \epsilon}\right] .
$$

In addition to these equations, the slow-roll evolution of the field $\phi$ is given by:

$$
\frac{d \phi / m_{P}}{d N_{e}}=-\frac{\sqrt{2 \epsilon}}{1+Q}
$$

For completeness, we also show the evolution of $Q$ :

$$
\frac{d Q}{d N_{e}}=\frac{Q}{1+Q+6 Q(1+\sigma)}\left[10\left(1+\frac{6}{5} \sigma\right) \epsilon-6(1+\sigma) \eta+8\left(1+\frac{3}{4} \sigma\right) \frac{m_{P}}{\phi} \sqrt{2 \epsilon}\right] .
$$

These results generalize the ones obtained in [40] for the case with no bulk viscosity. Note the difference in the notation between our $\sigma$ and the $\sigma_{\phi}$ defined there, which have replaced here by $\sqrt{2 \epsilon}\left(\phi / m_{P}\right)$. Nevertheless, as we have shown in (3.24), $|\sigma| \lesssim 1$ and then the results in [40] concerning whether the conditions increase or decrease during the evolution are still valid.

The last question before entering in the particular details of each model is how we are treating the bulk viscosity. In this Section we will use the non-causal description of the bulk viscosity, i.e. the Eckart theory, and place limits on the validity of this approximation. As discussed previously, the Eckart description is a good approximation for low values of $\Theta=\tau H$. The Hubble parameter is given by (2.7) and the relaxation time $\tau$ is obtained from Eq. (5.4). Using for example the first expression for the bulk viscosity in Eq. (5.2) (the second expression for the bulk viscosity 
in Eq. (5.2) can be easily seen as obtained from the first, when neglecting the temperature independent terms in $m_{y}(T)$ and in $\left.\tilde{m}_{y}\right)$, we obtain that

$$
\tau \approx 9.77 \times 10^{6} \frac{m_{y}^{3}(T)}{\lambda_{y}^{4} T^{4}}
$$

where for the superpotential (6.1), we have that $\lambda_{y}=6 h^{2}$. Based on the previous discussions, we consider the Eckart approximation to be valid when $\Theta \lesssim 10^{-2}$, which translates into (using that in the high-temperature limit $\left.m_{y}(T) \approx h T / 2\right)$

$$
h \gtrsim 10\left(\frac{H}{T}\right)^{1 / 5} .
$$

One of the conditions for warm inflation is that $T / H \gtrsim 1$, therefore, we can easily arrange the condition (6.9) to be satisfied when deep in the warm inflationary regime, particularly in the strong dissipative regime, which can also allow for perturbative values for the coupling $h$. The effect of including a causal description of the viscosity is to produce a lower value of the bulk viscous pressure than in the non-causal case with the same coefficient $C_{b}$. The consequence is a shift around the $5 \%$ level in the entire parameter spaces shown in the next subsections to higher values of $C_{b}$. This is explicitly verified below for the specific inflaton potential models we have studied.

\section{A. Chaotic model}

We first consider a chaotic inflation potential of the form:

$$
V(\phi)=\frac{\lambda}{4} \phi^{4}
$$

where we have used $\lambda=10^{-14}$. However this parameter is only relevant for the amplitude of the power spectrum, which we are not interested in here. Using again that $\Upsilon=C_{\phi} T^{3} / \phi^{2}$, for the potential (6.10) the slow-roll parameters, Eq. (3.15), are given by

$$
\eta=12\left(\frac{m_{P}}{\phi}\right)^{2}, \quad \epsilon=\frac{2}{3} \eta, \quad \beta=-\frac{2}{3} \eta .
$$

Therefore, the value of the field decreases during inflation, meanwhile, the dissipative ratio $Q$ and $T / H$ both increase. Hence, once the condition on $T>H$ is fulfilled initially, it is always satisfied. The ratio $\phi / T$ decreases, but we have checked that it always remains above 10 as long as the other conditions are fulfilled. The parameter $\epsilon_{H}$ increases during inflation and, as a consequence, warm inflation ends when $\epsilon_{H}=1$. Finally, the condition $\rho_{\phi}>\rho_{r}$ is controlled by the stability condition (3.26). It is only necessary to check that the stability condition is positive at the beginning of inflation, as it does not change sign during the evolution. This last statement is true for all the models studied.

The available parameter space is shown in Fig. 4. For completeness we have included the parameter space excluded for different values of the hydrodynamic condition $\left|\Pi / p_{r}\right|$, namely $0.1,0.5$ and 1 . We observe that the enlargement of the parameter space in regions of low $C_{\phi}$ is not very efficient. In particular, the minimum value of $C_{\phi}$ is reduced from $2.1 \times 10^{6}$ up to $1.6 \times 10^{6}$. These values have been confirmed by using the NLCDH description of the bulk viscosity, Eq. (2.27), with a constant $\tau$ fixed by imposing initially the values $\Theta=0.01,0.9$. The NLCDH description reduces the bulk viscous pressure associated to a $C_{b}$ value when $\Theta$ grows, which means that higher values of $C_{b}$ are allowed before the condition $\left|\Pi / p_{r}\right|$ is violated. However, at the same time, for the same value of $C_{\phi}$, higher values of $C_{b}$ are required to avoid the $T / H<1$ exclusion region, therefore the effects cancel each other and the minimum value of $C_{\phi}$ is independent of the initial value $\Theta$.

We have also found that the condition $\left|\Pi / p_{r}\right|$ is the most restrictive one in almost the full parameter space and that the instability regime studied in the previous sections is far beyond the limit imposed by this condition. In the analysis we have fixed the initial values such that the exclusion regions are the least stringent, that is, the upper region is as high as possible and the bottom one, as low as possible. We have fixed initial conditions in this way for the three models studied. 
In addition, we separate with black lines the regions where the dissipative ratio at horizon crossing $Q_{*}$ is always greater than one from the regions where it is always less than one. In the region between them, $Q_{*}$ can be either greater or less than one, depending on the initial value of $\phi$. In the regions that were not allowed in the absence of bulk viscosity, its main role is to produce enough e-folds of inflation. In regions allowed with no bulk viscosity, the total number of e-folds is increased. We quantify this effect in terms of the percentage difference $\Delta N_{e}$, defined as

$$
\Delta N_{e}=\frac{N_{e}^{\text {bulk }}-N_{e}^{\text {no-bulk }}}{N_{e}^{\text {no-bulk }}} \times 100,
$$

where $N_{e}^{\text {bulk }}$ is the maximum number of e-folds obtained with bulk viscosity for a certain $C_{\phi}$ and $N_{e}^{\text {no-bulk }}$ is the equivalent without bulk viscosity. The results are shown in Fig. 5 .

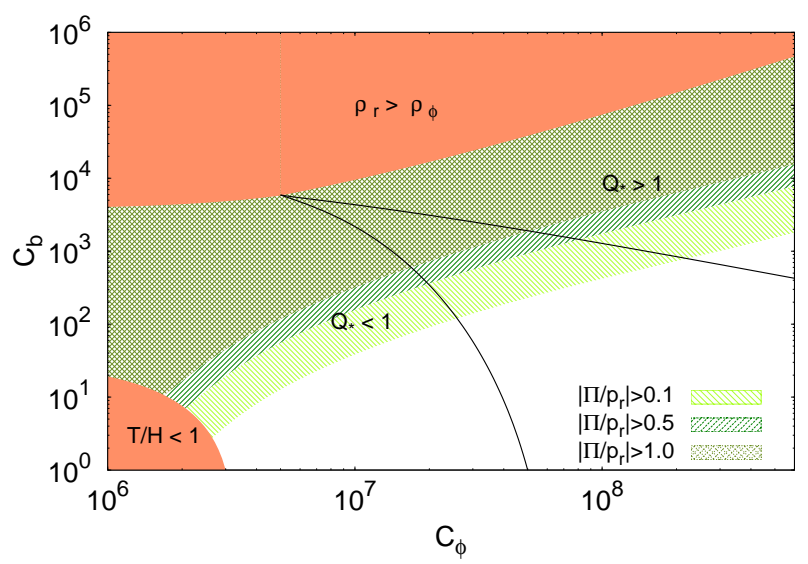

FIG. 4: Parameter space for the chaotic model. The green regions are excluded because of the violation of the condition written in the plot. The lines separate regions where $Q_{*}<1$ and $Q_{*}>1$ respectively. In the region between them, we can have both $Q_{*}<1$ and $Q_{*}>1$ for different values of $\phi(0)$.

The bulk viscosity can enhance the number of e-folds through two mechanisms. The first one is, for a given initial value of the field, to reduce the redshift of the radiation energy density by decreasing its total pressure. This effect produces an increase in the value of $Q$, which goes as $\rho_{r}^{3 / 4}$ and is related to the number of e-folds via

$$
N_{e}=\int_{\phi_{\text {end }}}^{\phi(0)} \frac{3 H^{2}(1+Q)}{V_{, \phi}} d \phi,
$$

where $\phi(0)$ is the initial value of the field and $\phi_{\text {end }}$, the value of the field at the end of inflation. Hence, the increase in $Q$ leads to a larger number of e-folds. However, we have checked that this mechanism is subdominant in the quartic potential, providing an efficiency up to $2 \%$.

The second mechanism allows to increase the initial value of the field. From Eq. 6.13) it can be seen that this produces more e-folds by increasing the integration interval. In the absence of bulk viscosity, there is an upper limit on the value of the field because of the condition $T / H>1$. As the bulk viscosity increases the value of $T$, it pushes upwards this limit and, hence, it is possible to use larger values of the field. However, there is a bound to this effect, provided by the condition $\left|\Pi / p_{r}\right|<1$, which translates into a new upper limit to $\phi(0)$. The decrease in this effect shown in Fig. [5 is because the limits on the initial value of the field grow at different rates with $C_{\phi}$, with the former growing faster, which causes the difference on the limits to decrease with $C_{\phi}$ and this, in turn, reduces the difference between the viscous and non-viscous cases. From Eq. (6.4) we can obtain the value of $C_{b}$ that maximizes this mechanism. Using the values $\left|\Pi / p_{r}\right|=1, T / H=1$ and $g_{*}=225.78$ as an example, we find that $C_{b}=8.25$. This argument is model independent, so that we find the same value of $C_{b}$ in the three models studied and independently of the value of $C_{\phi}$.

\section{B. Hybrid models}

We consider now small field models of inflation with an inflationary potential given by: 


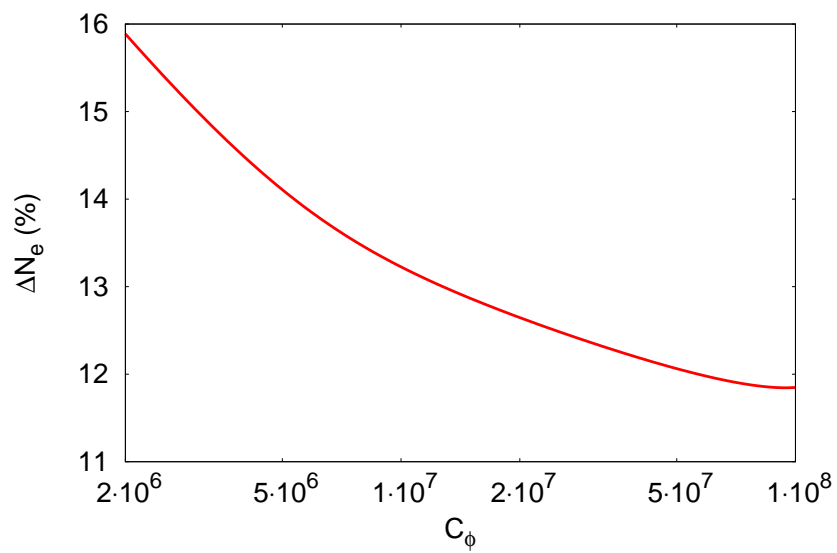

FIG. 5: Maximum enhancement in the number of e-folds for different values of $C_{\phi}$ for the model with quartic potential. The maximum value is obtained for $C_{b}=8.25$.

$$
V=V_{0}\left[1+\frac{\delta}{2}\left(\frac{\phi}{m_{P}}\right)^{2}\right],
$$

where $V_{0}$ is the scale and $\delta$ a model parameter. Here we have used $V_{0}=10^{-8} m_{P}^{4}$ and study the dynamics for two representative values for the parameter $\delta$. The slow-roll parameters, in the case of the inflaton potential given by Eq. (6.14), are now given by

$$
\eta=\delta, \quad \epsilon=\frac{\delta^{2}}{2}\left(\frac{\phi}{m_{P}}\right)^{2}, \quad \beta=-2 \eta
$$

During the evolution the value of $\phi$ decreases, while the value of $Q$ increases. The evolution of the value of $T / H$ depends on the value of Q: for $Q<1, T / H$ increases and for $Q>1$, it decreases; $\phi / T$ always decreases, and $\epsilon_{H}$ is not relevant in this model because it is suppressed by a factor $\left(\phi / m_{P}\right)^{2}$, which is usually very small due to the fact that $\phi \ll m_{p}$. Hence, $\epsilon_{H}$ is always below one. As a result, inflation ends because the conditions imposed either on $T / H$ or $\phi / T$ are violated, or because the field reaches its critical value.

In Fig. 66, the parameter space of the hybrid models with $\delta=0.1$ (left panel) and $\delta=10$ (right panel) are plotted. In the left panel, as in the previous model, the black lines separate regions with different value of the dissipative ratio at horizon crossing. However, in the right panel, the dissipative ratio is always above one. For a higher value of $\delta$ we can maintain $\eta /(1+Q)$ below one only for $Q_{*}>1$. In the left panel the bottom excluded region is forbidden for the same reason as in the chaotic model. Nevertheless, in the right panel we find that the excluded region is forbidden by the $\phi / T>10$ condition. This is caused again by the higher value of $\eta$. The parameter $\delta$ in Eq. (6.14) measures the curvature of the potential. Thus, for higher values of $\delta$ the field evolves faster. As a result, the condition on $\phi / T$ is reached first than in the case for smaller values for the parameter $\delta$.

The minimum value of $C_{\phi}$ in this case of inflation with the hybrid type of potential, Eq. (6.14), is reduced from $3.5 \times 10^{4}$ up to $2.6 \times 10^{4}$, for $\delta=0.1$, while for $\delta=10$ it is reduced from $5 \times 10^{4}$ up to $4.1 \times 10^{4}$. Making use of the $N L C D H$ description of the bulk with a constant $\tau$ fixed by imposing the initial values $\Theta=0.001$, 0.9 , we found that the minimum value $C_{\phi}$ is independent of the initial choice of $\Theta$. The effect on the number of e-folds is shown in Fig. 7. As in the previous case, the constant field mechanism is subdominant, with an efficiency of around a $3 \%$ for the $\delta=10$ case and a $6 \%$ efficiency for the $\delta=0.1$ case.

\section{Hilltop models}

We now consider new inflation hilltop type of models, which are characterized by an inflaton potential given by

$$
V=V_{0}\left[1-\frac{|\delta|}{2}\left(\frac{\phi}{m_{P}}\right)^{2}\right]+\cdots,
$$




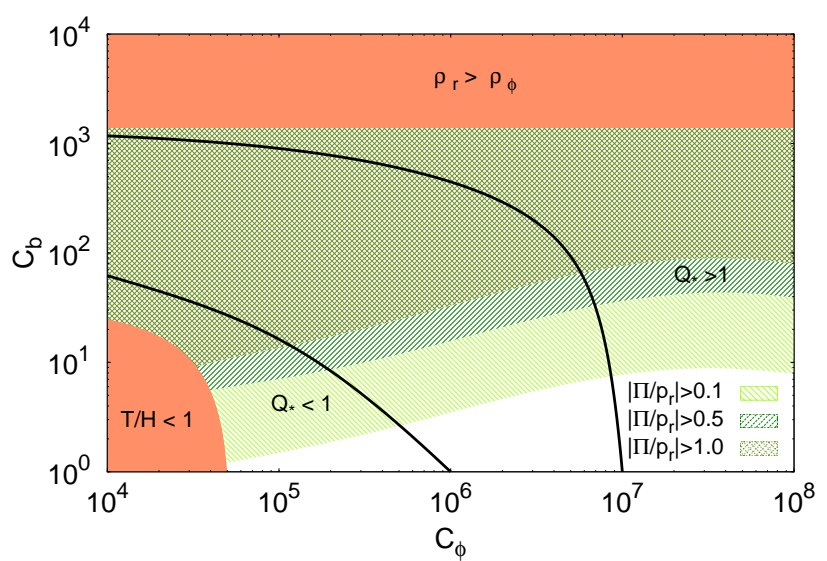

(a) $\delta=0.1$

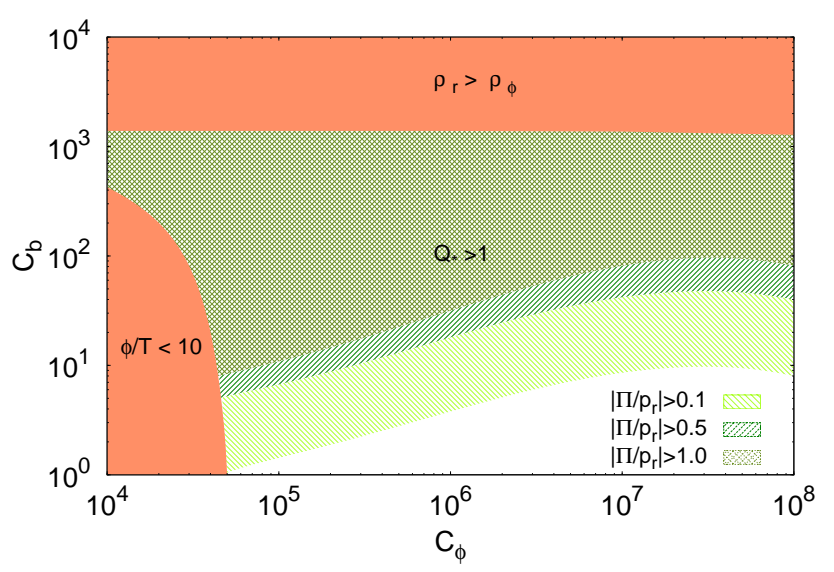

(b) $\delta=10$

FIG. 6: Parameter space for the hybrid models with $\delta=0.1,10$ The green regions are excluded because of the violation of the condition written in the plot. In the left plot the lines separate regions where $Q_{*}<1$ and $Q_{*}>1$ respectively. In the region between them, we can have both $Q_{*}<1$ and $Q_{*}>1$ for different values of $\phi(0)$. In the right plot, $Q_{*}$ is always above 1 .

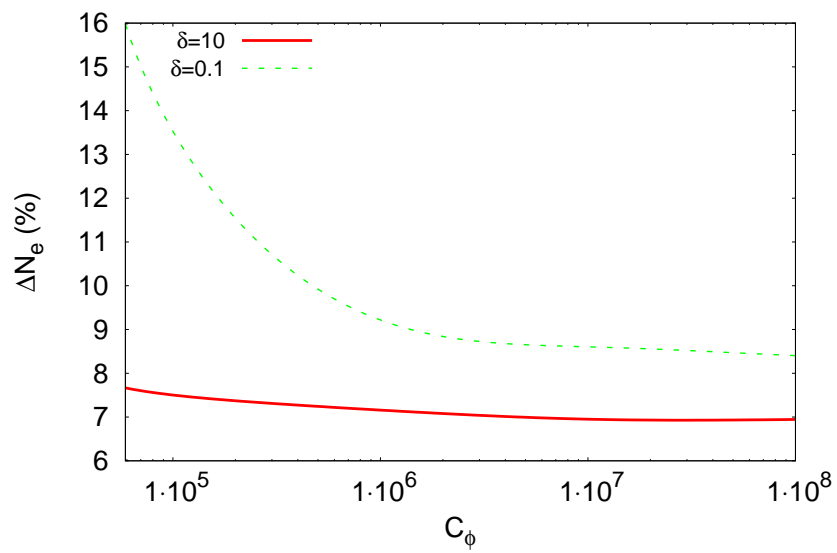

FIG. 7: Maximum enhancement in the number of e-folds for different values of $C_{\phi}$ in the case of the inflaton potential given by Eq. (6.14). The maximum value is obtained for $C_{b}=8.25$.

where the dots account for higher-order terms and $V_{0}=10^{-8} m_{P}^{4}$. This is a potential similar to the two previous ones, but with a negative squared inflaton mass. The slow-roll parameters are still given by those in Eq. (6.15), but with the change $\delta \rightarrow-\delta$. In these models $\phi, \phi / T, T / H$ and $\epsilon_{H}$ increase during the evolution, while $Q$ decreases. Inflation ends when the field reaches a large enough value, so that higher-order terms in the potential start contributing and $\epsilon_{H}$ becomes greater than one.

The parameter space for the hilltop model Eq. (6.16) is shown in Fig. 8, for the cases of $\delta=0.1$ (left panel) and $\delta=1$ (right panel). Once again, in the left panel the black curves separate regions with $Q_{*}$ greater or less than one at horizon crossing, and in the right panel, the dissipative ratio at horizon crossing is always greater than one due to the large value of $\eta$.

Now, the minimum value of $C_{\phi}$ is reduced from $3.3 \times 10^{4}$ up to $2.5 \times 10^{4}$ for $\delta=0.1$ and from $5.9 \times 10^{4}$ up to $5.1 \times 10^{4}$ for $\delta=1$. We have checked these values with the NLCDH description of the bulk with a constant $\tau$ fixed by imposing the initial values $\Theta=0.001,0.9$. The effect on the number of e-folds is shown in Fig. 9. As in the other cases studied, the constant field mechanism is subdominant with efficiencies around a $5 \%$ and a $3 \%$ for the $\delta=1$ and the $\delta=0.1$ cases respectively. The initial value mechanism works reversely compared with the previous potentials. Here it allows to use lower initial values of the field, however, as the value of the field grows in this case, this reduction implies an increase of the integration interval in Eq. (6.13). In addition, note that in this potential, the lower the value of the field, the larger the value of $H$ and thus, the lower is the ratio $T / H$. This argument also applies to the 
$\left|\Pi / p_{r}\right|$ condition, therefore, there are lower limits to $\phi(0)$ rather than upper ones.

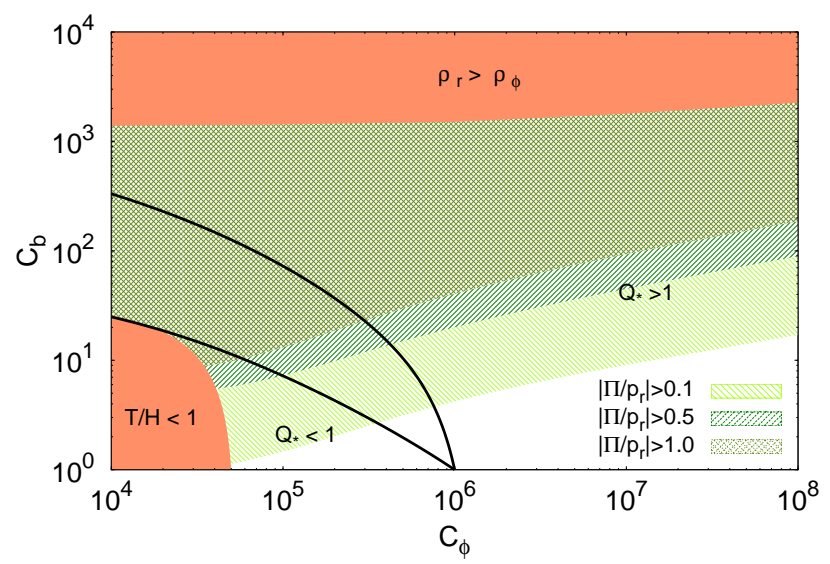

(a) $\delta=0.1$

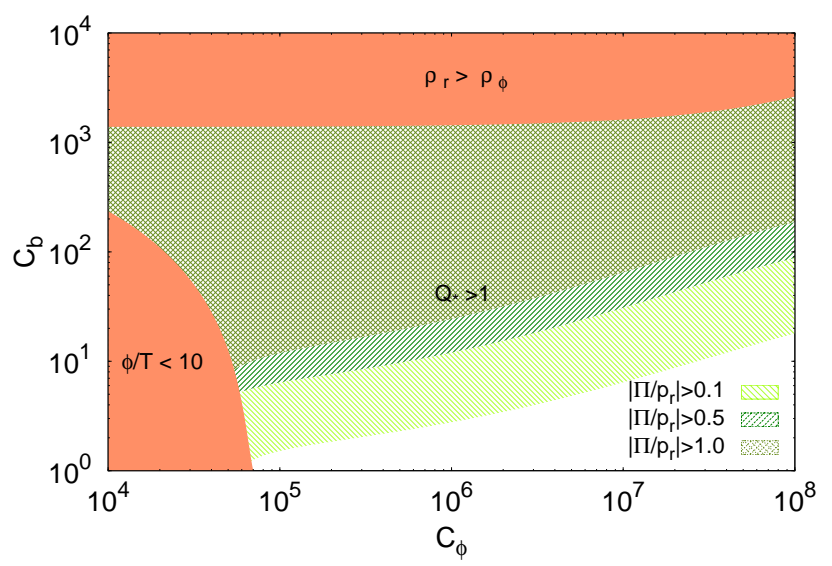

(b) $\delta=1$

FIG. 8: Parameter space for the hilltop models with $\delta=0.1,1$. The green regions are excluded because of the violation of the condition written in the plot. In the left plot the lines separate regions where $Q_{*}<1$ and $Q_{*}>1$ respectively. In the region between them, we can have both $Q_{*}<1$ and $Q_{*}>1$ for different values of $\phi(0)$. In the right plot, $Q_{*}$ is always above 1 .

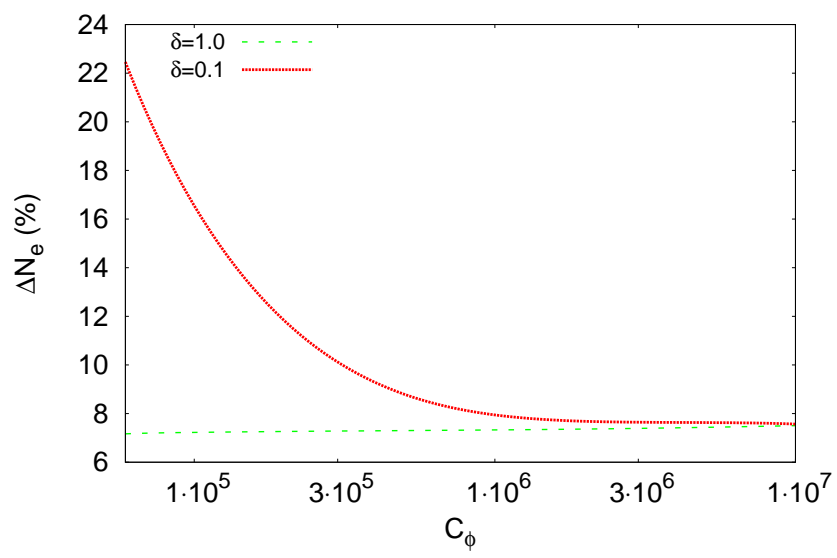

FIG. 9: Maximum enhancement in the number of e-folds for different values of $C_{\phi}$, for the case of the inflaton potential Eq. (6.16). The maximum value is obtained for $C_{b}=8.25$.

\section{The bulk viscosity coefficient}

Let us now briefly discuss the expected values for the bulk viscosity coefficient for a warm inflation model described by Eq. (6.1). In the context of the model described by Eq. (6.1), for the relevant temperature regime for warm inflation, characterized by $m_{\chi}>T$ and $m_{y} \ll T$, the dominant contribution to the bulk viscosity comes from the radiation thermal bath composed of the high-temperature particles, i.e. the $y$ particles and given by Eq. (5.2). We can see from the expression for the bulk viscosity Eq. (5.2) that the larger values for the bulk coefficient $C_{b}=\zeta_{b} / T^{3}$ appears in the intermediate temperature regime, $m_{y} \ll T \ll m_{y} / \lambda_{y}$, where the $y$ particles are already in the hightemperature regime, but the temperature is still not too high, such that in the thermal mass $m_{y}(T)$ the temperature corrections are subdominant. In this case, neglecting the thermal corrections to the mass, we get the estimate for $C_{b}$,

$$
C_{b} \approx 5.5 \times 10^{4} \frac{1}{\lambda_{y}^{4}} \frac{m_{y}^{6}}{T^{6}} \ln ^{2}\left(1.2465 m_{y} / T\right)
$$


recalling that for the model (6.1), $\lambda_{y}=6 h^{2}$. Taking $m_{y} / T \sim 0.1$, we obtain $70 \lesssim C_{b} \lesssim 1.8 \times 10^{4}$, for values of $h$ between 0.1 and 0.2. This is in the absence of further decay modes, which would increase even more the estimates for $C_{b}$ (the bulk viscosity coefficient is proportional to the radiation bath field degeneracy). These values are already within the window of values of the viscosity coefficient observed by the results in Figs. 4, 6] and 8, which allows warm inflation with smaller dissipation as a consequence of including a bulk viscous pressure.

\section{CONCLUSIONS}

In this work we have examined the stability of the dynamics of warm inflation when bulk viscous effects are included. The noncausal theory for the bulk viscous pressure, given by the Eckart hydrodynamics theory, was studied along with two other causal theories, the IS linear theory and the NLCDH theory, which is a simpler causal theory for the bulk viscosity, yet nonlinear. The stability of the resulting dynamical system for warm inflation has been studied before by the authors of Refs. [20], in the absence of bulk viscous pressure and [26], by including the effect of a viscous radiation bath for only the Eckart case. In this work we have extended these previous works by also carrying out the analysis of stability for the causal theories. We have also explored model building in the context of warm inflation with bulk viscous pressure.

By including the analysis of stability for the causal theories of IS and NLCDH, it becomes possible to clarify on the differences between these two theories and with that of the noncausal Eckart theory. We have seen significant differences for the radiation production in each of these different theories as the relaxation time of the radiation fluid increases. Among the three theories for the bulk pressure we have studied, the NLCDH case proved to be the most robust of them as far as stability is concerned. We have shown that it allows for relatively larger bulk viscous pressures as compared to the Eckart and IS cases. The noncausal theory is the one that most quickly can develop instabilities. Our results also suggest the significance and differences there might be in other nonlinear generalizations of theories for the viscous hydrodynamics of radiation fluids in cosmology, when relatively larger relaxation times are involved, but still within the range of validity of thermal equilibrium for the radiation bath, $\Theta<1$. Recalling that the Eckart and the IS theories have a long history of use in cosmology in general [22, 23], other nonlinear realizations of viscous hydrodynamics, in particular for the bulk pressure, may lead to significant differences in results, similar to the comparisons we found here for our problem.

In regards model building, we have shown that accounting for bulk viscous pressure effects in the radiation fluid can relax the requirements on the magnitude of the dissipation coefficient for the inflaton field, especially for a large bulk viscosity coefficient. This range of bulk viscosity coefficients can be realized within the regime of stability requirements in warm inflation, and this range is within reach of realistic model parameters. The upshot is, accounting for bulk viscosity effects makes warm inflation more robust. In particular warm inflation is more readily realized and with less constraints when bulk viscosity effects are accounted for versus when they are ignored. Although our examination of warm inflation was only for one particular form of the dissipative coefficient, we believe this conclusion will hold in general. Our results point to the importance of considering viscous effects in future studies of warm inflation. We also believe our results can have more general implications for systems involving viscous radiation fluids, in particular for cosmology. Very little has been discussed in the literature on the significance to cosmology of actual expressions of bulk viscosity computed from underlying particle physics models. This is one of the few papers that has examined this question for the specific case of warm inflation dynamics. However the methodology developed here can easily be extended to examine the same sorts of bulk viscosity effects in other relevant cosmological epochs, such as during the reheating phase after inflation in cold inflation dynamics and during the radiation and matter dominated expansion regimes.

\section{Acknowledgments}

M.B-G. and R.C. are partially supported by MICINN (FIS2010-17395) and "Junta de Andalucía" (FQM101) A.B. acknowledges support from the STFC. R.O.R is partially supported by research grants from Conselho Nacional de Desenvolvimento Científico e Tecnológico (CNPq) and Fundação Carlos Chagas Filho de Amparo à Pesquisa do Estado do Rio de Janeiro (FAPERJ). G.S.V. is supported by Coordenação de Aperfeiçoamento de Pessoal de Nível Superior (CAPES). R.O.R. and G.S.V. would also like to thank T. Koide for discussions concerning the hydrodynamical 
equations with bulk viscosity.

[1] A. H. Guth, Phys. Rev. D 23 (1981) 347; K. Sato, Phys. Lett. B 99 (1981) 66; A. Albrecht and P. J. Steinhardt, Phys. Rev. Lett. 48 (1982) 1220; A. D. Linde, Phys. Lett. B 108 (1982) 389.

[2] A. D. Linde, Phys. Lett. B 129 (1983) 177.

[3] A. Berera and L. Z. Fang, Phys. Rev. Lett. 74 (1995) 1912 arXiv:astro-ph/9501024.

[4] A. Berera, Phys. Rev. Lett. 75 (1995) 3218; arXiv:astro-ph/9509049. Phys. Rev. D 54 (1996) 2519; arXiv:hep-th/9601134. Phys. Rev. D 55 (1997) 3346. arXiv:hep-ph/9612239.

[5] L. Z. Fang, Phys. Lett. B 95 (1980) 154 ; I. G. Moss, Phys. Lett. B 154 (1985) 12; J. Yokoyama and K. I. Maeda, Phys. Lett. B 207 (1988) 31.

[6] E. A. Calzetta and B.-L. B. Hu, Nonequilibrium Quantum Field Theory (Cambridge University Press, Cambridge, 2008).

[7] A. Berera, M. Gleiser, R. O. Ramos, Phys. Rev. D 58 (1998) 123508. arXiv:hep-ph/9803394[hep-ph]].

[8] A. Berera, I. G. Moss and R. O. Ramos, Rept. Prog. Phys. 72 (2009) 026901. [arXiv:0808.1855 [hep-ph]].

[9] D. Boyanovsky, D. Cormier, H. J. de Vega and R. Holman, Phys. Rev. D 55 (1997) 3373 hep-ph/9610396. D. Boyanovsky, D. Cormier, H. J. de Vega, R. Holman and S. P. Kumar, Phys. Rev. D 57 (1998) 2166 hep-ph/9709232.

[10] M. Gleiser and R. O. Ramos, Phys. Rev. D 50 (1994) 2441 hep-ph/9311278.

[11] N. Barnaby, E. Pajer and M. Peloso, Phys. Rev. D 85 (2012) 023525, [arXiv:1110.3327 [astro-ph.CO]]; D. Lopez Nacir, R. A. Porto, L. Senatore and M. Zaldarriaga, JHEP 1201 (2012) 075, arXiv:1109.4192 [hep-th]].

[12] A. Berera, M. Gleiser, R. O. Ramos, Phys. Rev. Lett. 83 (1999) 264. arXiv:hep-ph/9809583.

[13] M. Bastero-Gil, A. Berera and R. O. Ramos, JCAP 1109 (2011) 033. [arXiv:1008.1929 [hep-ph]].

[14] M. Bastero-Gil, A. Berera, R. O. Ramos and J. G. Rosa, arXiv:1207.0445 [hep-ph]].

[15] I. G. Moss and C. Xiong, arXiv:hep-ph/0603266].

[16] A. Berera, R. O. Ramos, Phys. Rev. D 63 (2001) 103509. arXiv:hep-ph/0101049.

[17] A. Berera and R. O. Ramos, Phys. Lett. B 567 (2003) 294 arXiv:hep-ph/0210301.

[18] A. Berera and R. O. Ramos, Phys. Lett. B 607 (2005) 1 arXiv:hep-ph/0308211]; Phys. Rev. D 71 (2005) 023513 arXiv:hep-ph/0406339.

[19] L. M. H. Hall and I. G. Moss, Phys. Rev. D 71 (2005) 023514 arXiv:hep-ph/0408323.

[20] I. G. Moss and C. Xiong, JCAP 0811 (2008) 023 [arXiv:0808.0261 [astro-ph]].

[21] S. Weinberg, Gravitation and Cosmology, (New York, NY, Wiley, 1972).

[22] G. L. Murphy, Phys. Rev. D 8 (1973) 4231; Y. B. Zeldovich, Pisma Zh. Eksp. Teor. Fiz. 12 (1970) 443; B. L. Hu, Phys. Lett. A 90 (1982) 375; I. Waga, R. C. Falcao and R. Chanda, Phys. Rev. D 33 (1986) 1839; R. Maartens, Class. Quant. Grav. 12 (1995) 1455; W. Zimdahl, D. Pavon and J. Triginer, Helv. Phys. Acta 69 (1996) 225 arXiv:astro-ph/9608060. W. Zimdahl and D. Pavon, Phys. Rev. D 61 (2000) 108301; M. Giovannini, Class. Quant. Grav. 22 (2005) 5243 arXiv:astro-ph/0504655]; S. del Campo, R. Herrera, D. Pavon, Phys. Rev. D 75 (2007) 083518 arXiv:astro-ph/0703604.

[23] J. -S. Gagnon and J. Lesgourgues, JCAP 1109 (2011) 026 arXiv:1107.1503 [astro-ph.CO]]; I. Brevik, E. Elizalde, S. Nojiri and S. D. Odintsov, Phys. Rev. D 84 (2011) 103508 arXiv:1107.4642 [hep-th]]; O. F. Piattella, J. C. Fabris and W. Zimdahl, JCAP 1105 (2011) 029 arXiv:1103.1328 [astro-ph.CO]]; C. Bogdanos, A. Dimitriadis and K. Tamvakis, Phys. Rev. D 75 (2007) 087303 arXiv:hep-th/0611094.

[24] S. Jeon, Phys. Rev. D 52 (1995) 3591 arXiv:hep-ph/9409250; S. Jeon and L. G. Yaffe, Phys. Rev. D 53 (1996) 5799 arXiv:hep-ph/9512263.

[25] H. P. de Oliveira and R. O. Ramos, Phys. Rev. D 57 (1998) 741 arXiv:gr-qc/9710093.

[26] S. del Campo, R. Herrera, D. Pavon and J. R. Villanueva, JCAP 1008 (2010) 002 [arXiv:1007.0103 [astro-ph.CO]].

[27] J.P. Mimoso, A. Nunes and D. Pavon Phys. Rev. D 73 (2006) 023502 arXiv:gr-qc/0512057.

[28] C. Eckart, Phys. Rev. 58 (1940) 919.

[29] W. Israel, Ann. Phys. (N.Y.) 100 (1976) 310.

[30] W. Israel, J. Stewart, Ann. Phys. (N.Y.) 118 (1979) 341.

[31] G. S. Denicol, T. Kodama, T. Koide and P. .Mota, J. Phys. G 36 (2009) 035103. arXiv:0808.3170 [hep-ph]]

[32] R. Maartens and V. Mendez, Phys. Rev. D 55 (1997) 1937 arXiv:astro-ph/9611205.

[33] P. Ilg and H. C. Ottinger, Phys. Rev. D 61 (2000) 023510.

[34] M. Bastero-Gil, A. Berera and R. O. Ramos, JCAP 1107 (2011) 030 arXiv:1106.0701 [astro-ph.CO]].

[35] R. Maartens, arXiv:astro-ph/9609119; R. Maartens, J. Triginer, Phys. Rev. D56 (1997) 4640 arXiv:gr-qc/9707018.

[36] W. A. Hiscock and L. Lindblom, Annals Phys. 151 (1983) 466.

[37] L. Landau, E.M. Lifshitz, Fluid mechanics (Addison Wesley, Reading, MA, 1958).

[38] A. R. Liddle, P. Parsons and J. D. Barrow, Phys. Rev. D 50 (1994) 7222 arXiv:astro-ph/9408015.

[39] R. O. Ramos and L. A. Silva, work in progress.

[40] M. Bastero-Gil, A. Berera, Int. J. Mod. Phys. A 24 (2009) 2207 arXiv:0902.0521 [hep-ph]].

[41] P. Chakraborty and J. I. Kapusta, Phys. Rev. C 83 (2011) 014906 arXiv:1006.0257 [nucl-th]].

[42] E. Komatsu et al., Astrophys. J. Suppl. 192 (2011) 18 arXiv:1001.4538 [astro-ph.CO]].

[43] D. Boyanovsky, C. Destri, H. J. De Vega and N. G. Sanchez, Int. J. Mod. Phys. A 24 (2009) 3669 arXiv:0901.0549 [astro-ph.CO]]. 